\title{
Nach dem permissiven Konsens. Das Demokratiedefizit der Europäischen Union
}

\section{Einleitung ${ }^{1}$}

Für Bundeskanzlerin Merkel war die Sache klar. Um den Bedürfnissen der Bürger besser gerecht zu werden, müsse Europa transparenter und handlungsfähiger werden, sagte sie in ihrer Regierungserklärung vom 10. Mai 2006. Die „Neubegründung" der Europäischen Union liege im Nachweis politischer Gestaltbarkeit in einer sich ändernden Welt. Nur ein Europa, das die Sorgen der Menschen ernst nehme und drängende Probleme löse, könne verlorenes Vertrauen zurückgewinnen. Wohl wahr. Doch sind in der EU Transparenz und Handlungsfähigkeit tatsächlich ein Gespann, das in dieselbe Richtung zieht? Lange Zeit galt, dass die Bevölkerung mit freundlicher Indifferenz auf die Resultate blickte, die das nationale Führungspersonal in Brüssel aushandelte. Eine direkte Beteiligung war unnötig, solange eine breite Mehrheit mit den Ergebnissen übereinstimmte. Die europäische Einigung wurde durch einen „permissiven Konsens“ (Lindberg/Scheingold 1970, S. 41) getragen. Allem Anschein nach erodiert diese stillschweigende Zustimmung.

Aus den negativen Voten zum Verfassungsentwurf in Frankreich und den Niederlanden spricht politisches Unbehagen am gegenwärtigen Stand der Integration: Marktöffnung und Konkurrenz empfinden viele Bürger als Bedrohung; den eigenen Einfluss auf europäische Entscheidungen schätzen sie gering ein; und die Funktionsweise sowohl der nationalen als auch der EU-Demokratie beurteilen sie skeptisch. Vor diesem Hintergrund liegt die Frage nahe, wie die EU demokratischer und bürgernäher werden kann. Aber längst nicht alle Beobachter teilen dieses Anliegen. In der EU-Forschung wird darüber diskutiert, ob es das häufig beklagte Demokratiedefizit überhaupt gibt und, falls doch, ob es behoben werden sollte. In dieser Auseinandersetzung argumentiert die eine Seite, dass mehr Transparenz die Handlungsfähigkeit der Politik verringere, während die andere mehr Bürgerbeteiligung einfordert, um die Legitimitätskrise der EU zu überwinden.

Mein Aufsatz schlägt ein Analyseraster vor, das zeigen will, wie die Konfliktlinien

1 Für hilfreiche Kommentare danke ich Michael Blauberger, Holger Döring, Martin Höpner, Alexander Kuo, Ines Läufer, Simone Leiber, Dirk Leuffen, Wendelmoet van den Nouland und Thomas Zittel. 
in der Debatte um das Demokratiedefizit der EU verlaufen. Vier Sichtweisen bestimmen gegenwärtig die Diskussion: (1) Die optimistische Position: Eine Aufwertung demokratischer Verfahren in der EU sei nicht nur erstrebenswert, sondern auch möglich. Vertreter dieser Sichtweise unterbreiten institutionelle Reformvorschläge, die die EU an nationalstaatliche Formen der Politik heranführen sollen. (2) Die Pessimisten: Eine Demokratisierung der EU sei zwar wünschenswert, aber auf absehbare Zeit unmöglich. Vertreter dieser Gruppe sehen im Fortgang der Integration eine Gefahr vor allem auch für die nationale Demokratie. (3) Die Apologeten bestreiten das EU-Demokratiedefizit. Sie sehen die Reformvorschläge der Optimisten als realisierbar an, lehnen sie aber ab, weil die EU ihre Handlungsfähigkeit verlöre. Der Vorteil delegierter Entscheidungen liegt aus dieser Sicht darin, Entscheidungen aus nationalen Verteilungskämpfen herauszuhalten und dem Zugriff von Interessengruppen zu entziehen. Schließlich (4) geht die fatalistische Position davon aus, dass eine Demokratisierung der EU weder möglich noch erstrebenswert sei. Die EU sei wohlweislich ein bürokratischer konsoziativer Staat, in dem nationale Eliten Kompromisse aushandeln. Mehr Bürgerbeteiligung und Transparenz würden das Verhandlungsgleichgewicht stören.

Abbildung 1: Die vier Positionen zum EU-Demokratiedefizit

\begin{tabular}{|c|c|c|c|}
\hline \multirow{4}{*}{$\begin{array}{l}\text { Demokratisierung } \\
\text { erstrebenswert }\end{array}$} & & \multicolumn{2}{|c|}{$\begin{array}{l}\text { Demokratisierung } \\
\text { möglich }\end{array}$} \\
\hline & & ja & nein \\
\hline & ja & OPTIMISTEN & PESSIMISTEN \\
\hline & nein & APOLOGETEN & FATALISTEN \\
\hline
\end{tabular}

\section{Darstellung der vier Positionen}

Die nächsten vier Abschnitte ordnen den vier Grundpositionen einzelne Autoren zu. Ihre Sichtweisen unterscheiden sich sowohl in ihrem Verständnis der EU als auch in den daraus abgeleiteten Empfehlungen. Während die einen die Demokratisierung der Europäischen Union erhoffen, betonen die anderen die Risiken zunehmender Politisierung und bezweifeln die Existenz eines Legitimitätsmangels der EU-Politik. Dabei ist zu beachten, dass die Autoren selbst die Begriffe - Optimisten, Pessimisten, Apologeten und Fatalisten - nicht benutzen und möglicherweise auch mit der Zuordnung nicht einverstanden wären. Dennoch gibt diese Typologie Aufschluss über verschiedene Perspektiven auf die EU-Politik und die mit der europäischen Integration verbundenen Herausforderungen für die Demokratie. 


\subsection{Die Optimisten}

Die Europaforschung hat sich in den vergangenen Jahren verstärkt Einsichten der vergleichenden Politikwissenschaft zu Eigen gemacht. Begreift man die EU als politisches System, können Mechanismen der Präferenzaggregation und der Entscheidungsfindung mit denen anderer politischer Systeme verglichen werden. Dabei wird deutlich, dass die EU in manchen Politikfeldern „Staatsqualität" erreicht, während sie in anderen einer internationalen Organisation gleicht. Diese Perspektive löst sich von der älteren Integrationstheorie, die vorwiegend nach den Ursachen der Vergemeinschaftung suchte. Stattdessen steht die Frage im Vordergrund, wie das politische System der EU kollektiv bindende Entscheidungen hervorbringt. Im Zentrum des Interesses steht das Zusammenspiel der EU-Institutionen. So untersuchen beispielsweise Tsebelis und Garrett (2001) mit Hilfe des Vetospieler-Ansatzes den Einfluss der EU-Institutionen bei unterschiedlichen Entscheidungsverfahren auf die Gesetzgebung. Das Instrumentarium unterscheidet sich dabei nicht von dem, das auch in der Analyse nationaler politischer Systeme eingesetzt wird.

Besondere Aufmerksamkeit hat die vergleichende Politikwissenschaft dem Europäischen Parlament gewidmet. Dabei steht die Frage im Zentrum, ob sich die Abgeordneten im Abstimmungsverhalten nach Fraktionszugehörigkeit oder nach Herkunft unterscheiden. Falls die Herkunft das Verhalten der Abgeordneten bestimmt, verdoppelt das Parlament die zwischenstaatliche Ausrichtung des Ministerrats. Während im deutschen Föderalismus der Parteienwettbewerb nicht nur den Bundestag, sondern auch den Bundesrat dominiert, steht in der EU zu befürchten, dass die territoriale Logik beide Kammern beherrscht. An keiner Stelle fände dann ein Wettbewerb um konkurrierende Politikentwürfe statt. Doch die Forschung der letzten Jahre betont, dass die EP-Fraktionen sich hinsichtlich des Abstimmungsverhaltens und der ideologischen Differenzen zwischen den Fraktionen immer weniger von nationalen Parlamenten unterscheiden (Kreppel 2002; Hix/Noury/Roland 2005). ${ }^{2}$

Dem Europaparlament kommt besondere Bedeutung zu, weil in dem Maß, wie es ein normales Parlament wird, der Weg für die Ausweitung seiner Kontroll- und Gesetzgebungsbefugnisse geebnet wird. Die Optimisten sehen gegenwärtig eine Diskrepanz zwischen der Bedeutung der EU-Politik und dem Grad der mit ihr verbundenen Kontroversen. Während die Auseinandersetzung zwischen Regierungsmehrheit und Opposition die nationale Politik prägt, gibt es in der EU kein Schattenkabinett, das die Kritik an Kommissionsvorschlägen mit einer alternativen Programmatik verknüpft. Zwar wurde der Einfluss des Europaparlaments auf die Auswahl der Europäischen Kommission gestärkt - der Kommissionspräsident ist nicht länger gegen den Willen der stärksten EP-Fraktion durchsetzbar -, aber aus den Europawah-

2 Im Gegensatz dazu zeigt Höpner (2005) am Beispiel der Übernahmerichtlinie, dass bei Abstimmungen mit gravierenden Folgen für die Mitgliedstaaten die Herkunft der Parlamentarier weiterhin den Ausschlag gibt. 
len gehen keine Regierungsfraktionen hervor, die dauerhaft die Arbeit der Regierung unterstützen.

Die Bürger verfügen über zwei Einflusskanäle in der EU, doch die Art, wie diese Kanäle gegenwärtig genutzt werden, weist paradoxe Züge auf (Mair 2005, S. 9). Über nationale Parlamentswahlen können die Wähler beeinflussen, wie integrationsfreundlich der Europäische Rat ist. Europaskeptische Wähler sollten daher in nationalen Wahlen ihren Widerspruch gegen Vertiefung und Erweiterung ausdrücken, denn diese Weichenstellungen werden intergouvernemental vorgenommen. Dies geschieht jedoch nicht, weil innenpolitische Themen die Wahlentscheidung bestimmen. Der zweite Einflusskanal sind die Europawahlen. Hier können die Wähler die Politikgestaltung der EU in Bereichen beeinflussen, in denen ein Kompetenztransfer bereits stattgefunden hat. Bei der Ausformulierung konkreter Policies verfügt das EP über mehr Macht als bei den Konstitutionalisierungsschritten. Doch gerade bei Europawahlen schneiden europaskeptische Parteien besonders gut ab. Der Protest gegen „zuviel Europa“ artikuliert sich damit an der falschen Stelle. Im Ergebnis bedeutet dies, dass Wahlen wenig Einfluss auf die europäische Politik ausüben:

„The choices in both channels become increasingly irrelevant to the outputs of the system, and the behaviour and preferences of citizens constitute virtually no formal constraint on, or mandate for, the relevant policy-makers. Decisions can be taken by political elites with more or less free hand" (Mair 2005, S. 9).

Die Antwort der Optimisten auf dieses Paradox ist, die demokratischen Verfahren in der EU aufzuwerten (Follesdal/Hix 2005, S. 18-22). Der Kommissionspräsident als Kopf der Exekutive könne beispielsweise direkt gewählt werden. Ein alternativer Vorschlag lautet, die Auswahl der Kommissare aus den Händen nationaler Regierungen in die des Europaparlaments zu legen. Die Kommission wäre dann der Parlamentsmehrheit verpflichtet, und Europawahlen würden zu Entscheidungen über unterschiedliche Programme. Ein dritter Vorschlag möchte Referenden als regelmäßige Befragung der Wähler nutzen. Gegenwärtig bieten Referenden eine seltene Gelegenheit, zur europäischen Integration Stellung zu nehmen. Empirische Untersuchungen belegen, dass der Nebenwahlcharakter in Referenden weniger ausgeprägt ist und europäische Themen mehr Gewicht haben (Garry/Marsh/Sinnott 2005). Europaweite Volksabstimmungen könnten, so die Hoffnung, die Entstehung einer europäischen Öffentlichkeit fördern. Schließlich setzt ein vierter Vorschlag bei der Transparenz von Ratsentscheidungen an. Das Abstimmungsverhalten der Regierungsvertreter solle bekannt gegeben werden, um nationalen Parlamenten eine bessere Kontrolle der Exekutive zu ermöglichen. Mehr Transparenz und nachträgliche Kontrolle disziplinierten das Verhalten in Exekutivkartellen. Dies könne den Einflussverlust nationaler Parlamente auf die EU-Entscheidungen zumindest in Teilen verringern.

Die eine Reformstrategie der Optimisten besteht also darin, die EU zu einem parlamentarischen Regierungssystem mit zwei Kammern weiterzuentwickeln. Dane- 
ben gibt es diejenigen, die ihren Vorschlägen das Modell deliberativer Politik zugrunde legen. Das Demokratiedefizit der EU kann aus dieser Sicht nur behoben werden, indem EU-Entscheidungen für gesellschaftliche Gruppen zugänglich und Gegenstand öffentlicher Debatte werden (Eriksen/Fossum 2004, S. 446). Um mehr Akzeptanz bei den Bürgern zu finden, muss die EU die technokratisch-intergouvernementale Politikgestaltung zugunsten partizipativer Entscheidungsverfahren aufgeben (Neyer 2005, S. 378-379). Vertreter dieser Reformperspektive sehen Ansätze eines „deliberativen Supranationalismus" bereits in den Komitologie-Verfahren verwirklicht, mit denen der Rat die Arbeit der Kommission kontrolliert (Joerges/Neyer 1997). Interessengeleitete Verhandlungen sollen durch Argumentation und gemeinsame Problemlösung ersetzt werden. Besondere Hoffnung setzen einige Autoren auf die Offene Methode der Koordinierung, in der sie eine Form des „demokratischen Experimentalismus" sehen (Eberlein/Kerwer 2002). Die Vorteile im Vergleich zur Gemeinschaftsmethode werden in besseren Beteiligungschancen, im Respekt für nationale Unterschiede und im Potenzial für wechselseitiges Lernen gesehen (Zeitlin 2005, S. 448-449). Die deliberative Demokratietheorie erscheint aus dieser Sicht nicht bloß als kritischer Maßstab, an dem sich notwendig unvollkommene Verfahren messen lassen müssen, sondern als Verhandlungen überlegenes Politikmodell, dessen Verwirklichung möglich und erstrebenswert ist (Neyer 2003).

\subsection{Die Pessimisten}

Die Autoren des letzten Abschnitts begreifen die Europäische Union als ein politisches System, das durch institutionelle Reformen demokratisiert werden kann. Die Pessimisten teilen zwar das Anliegen der Optimisten, sind aber skeptisch, ob eine Demokratisierung der EU möglich ist. ${ }^{3}$ Nicht die vergleichende Politikwissenschaft informiert ihren Blick, sondern die historische Makrosoziologie. Vor dem Hintergrund der Staats- und Nationenbildung in Westeuropa wird gefragt, welche Auswirkungen die EU auf die Mitgliedstaaten hat. Im Nationalstaat beruhte die innere Befriedung durch Demokratisierung und Ausbau des Sozialstaats auf der Grenzziehung nach außen. Territoriale Grenzen schufen die Voraussetzung für die rechtliche Gleichheit der Staatsangehörigen und für Umverteilung zwischen ihnen (Flora 2000, S. 50). Die europäische Integration ist der am weitesten fortgeschrittene Versuch, räumliche Grenzen und die Mitgliedschaft in politischen Verbänden neu zu definieren. Die wirtschaftliche Integration destabilisiert das Verhältnis von kultureller Zugehörigkeit, Solidarität und Demokratie in den Mitgliedstaaten, ohne dass auf absehbare Zeit ein europäischer Ersatz möglich scheint (Bartolini 2000, S. 132).

Ausgangspunkt dieser Perspektive ist die Theorie Stein Rokkans, der die Entste-

3 In Deutschland vertritt Graf Kielmansegg (2003) diese Position. Auf die Diskussion um die "No-demos-These“ wird hier nicht ausführlich eingegangen. 
hung der europäischen Nationalstaaten untersuchte. ${ }^{4}$ Bartolini (2005) und Ferrera (2005) nutzen Rokkans theoretisches Rüstzeug für eine Bestandsaufnahme der EU. Sie sehen in der europäischen Integration den sechsten großen Entwicklungstrend des europäischen Staatensystems. Die fünf früheren Entwicklungsstufen waren Staatsbildung, Entstehung des Kapitalismus, Nationenbildung, Demokratisierung und schließlich der Aufbau des Sozialstaats (Bartolini 2005, S. 364, Kapitel 2). Auch wenn die Sequenz sich in einzelnen Regionen Europas unterschied, beschreiben die fünf Entwicklungsstufen die erfolgreiche Herausbildung des demokratischen Nationalstaats in Nordwesteuropa. Grenzziehung und interne Strukturierung - zwei zentrale Konzepte Rokkans - waren historisch eng miteinander verbunden (Ferrera 2005, S. 21). Erst die Begrenzung von Exit-Optionen, also die effektive Kontrolle territorialer Grenzen, kanalisierte Protest und Opposition im Staat und führte schließlich zur Institutionalisierung politischer Rechte. Territoriale Konsolidierung, kulturelle Identität und Demokratisierung begünstigten die gesellschaftliche Integration im Nationalstaat (Flora 2000, S. 22). Demokratie und Solidarität beruhten folglich auch auf dem Zwang verhinderter Abwanderung. Werden Grenzen durchlässig, können sich zur Subventionierung anderer angehaltene Gruppen den als Zumutung empfundenen Verpflichtungen des Nationalstaats entziehen. Der EUBinnenmarkt bietet mobilen Faktoren die Möglichkeit zu gehen und verstärkt die Macht der territorial nicht Gebundenen gegenüber denen, die bleiben müssen.

Empirisch untersucht Ferrera (2005, Kapitel 4) den Anpassungsdruck, der vom Binnenmarkt auf die nationalen Sozialstaaten wirkt. Als Folge der europäischen Integration, vor allem durch das Prinzip der Inländergleichbehandlung und die Dienstleistungsfreiheit, sind „halbsouveräne Wohlfahrtsstaaten“ entstanden, die die Definitionsmacht über den Status als Anbieter oder Nachfrager sozialer Leistungen in Teilen verloren haben (Leibfried/Pierson 1998). Durchlässige Grenzen bedrohen die nationalen Solidarsysteme:

„Social sharing builds on 'closure'. It presupposes the existence of a clearly demarcated and cohesive community, whose members feel that they belong to the same whole and that they are linked by reciprocity ties vis-à-vis common risks and similar needs. [...] European integration, on the contrary, rests on 'opening': on weakening or tearing apart those spatial demarcations and closure practices that nation states have built to protect themselves" (Ferrera 2005, S. 2).

In den sozialen Grundrechten, die der Verfassungsvertrag vorgesehen hatte, und in der Offenen Methode der Koordinierung sieht Ferrera (2005, S. 237-253) dennoch Keime einer EU-Sozialpolitik, die die destabilisierenden Folgen offener Märkte abfangen könnten. ${ }^{5}$ Doch hängt grenzüberschreitende Solidarität auch davon ab, dass

4 Für eine Einführung in Rokkans Theorie siehe Flora (2000).

5 Ferrera äußert sich nicht direkt zum Demokratiedefizit und würde sich vermutlich selbst nicht zu den Pessimisten zählen. Zudem sieht er die Möglichkeit einer neuen Wohlfahrtstaatsarchitektur in Europa. Seine historische Perspektive warnt allerdings vor der Erwartung schneller Erfolge und der Vorstellung, dass ein genuin europäischer Sozialstaat entstehen könnte. 
sich ein stärkeres Wir-Gefühl der Europäer - auch in Abgrenzung zu „Nicht-Europäern" - herausbildet. Rokkans Theorie lehrt, dass eine Gemeinschaft, die ihre Grenzen nicht definiert, sich schwer tun wird, auch nur eine schwache Identität auszubilden. Und so wird, wie Offe bemerkt (1998, S. 133), der Nationalstaat auf absehbare Zeit der größte Sozialverband bleiben, der Umverteilungsopfer zumutbar machen kann. Soll die EU mehr als ein geopolitisches Projekt sein, muss festgelegt werden, wo die geografische Grenze der Mitgliedschaft liegt.

Neben der Sozialpolitik untersucht eine Reihe von Arbeiten, wie sich die nationalen Parteiensysteme durch die EU-Mitgliedschaft verändern. Spiegelten sie lange Zeit den Verlauf von Konfliktlinien (cleavage structures) der Vergangenheit, begannen sich diese ab den 1960er Jahren aufzulösen (Lipset/Rokkan 1967). Die Parteiensysteme wurden für neue Wettbewerber durchlässiger, und die Parteienidentifikation der Wähler ließ langsam nach. Falls der europäischen Integration eine ähnliche Bedeutung wie früheren Entwicklungstrends zukommt, müsste sich dies in neuen Konfliktlinien niederschlagen. Empirisch gibt es bisher nur vereinzelte Hinweise, dass die EU-Politik zu Parteineugründungen oder zu Abspaltungen geführt hat (Andeweg 1995; Mair 2001). Stattdessen lässt sich die Position von Parteien zur EU aus bestehenden Konfliktlinien ableiten (Marks/Wilson 2000; Hooghe/Marks/Wilson 2004). Aufgrund des erheblichen Anteils europakritischer Wähler prognostizieren Eijk und Franklin (2004, S. 47), dass der „schlafende Riese“ EU zukünftig auch Gegenstand der innenpolitischen Auseinandersetzung wird. Populistische Parteien haben bereits das Mobilisierungspotenzial europaskeptischer Positionen erkannt (Taggart 1998).

Die „Entgrenzung“, als die sich die europäische Integration beschreiben lässt, wirkt auf die Mitgliedstaaten zurück und, so die These der Pessimisten, verändert nicht nur die Sozialstaaten, sondern auch die nationale Demokratie:

"The main thrust of my argument in this work is that the institutional design of the EU militates to date against any stable form of political structuring for its representative actors, while its growing political production tends to undermine national mechanisms of political representation and legitimation. Institutional democratization is, in principle, easy to achieve, but any institutional democratization without political structuring is potentially catastrophic, and political structures cannot be created without important advances in system-building that, for now, are completely non-existent" (Bartolini 2005, S. 408).

Obwohl institutionelle Reformen, wie sie die Optimisten einfordern, möglich sind, kann mit ihnen das strukturelle Demokratiedefizit nicht behoben werden (Bartolini 2000, S. 156). Verantwortlich ist die heutige Arbeitsteilung, bei der die EU-Institutionen für mehr Wettbewerb verantwortlich sind, während nationale Regierungen die unerwünschten Folgen der Vermarktlichung kompensieren sollen. Die Mitgliedstaaten treffen zentrale wirtschafts- und sozialpolitische Entscheidungen nicht mehr eigenständig. Da jedoch in der EU keine offene Auseinandersetzung um die richtigen Konzepte stattfindet, entsteht ein Politikdefizit. Der Kompetenztransfer an vermeintlich unpolitische Institutionen entwertet die nationale Politik. Als Weichen- 
stellung inszenierte Wahlkämpfe verbergen nicht, dass der Gestaltungsspielraum von Regierungen in vielen Feldern eingeschränkt ist. Demokratie ist aber darauf angewiesen, dass wechselnde Mehrheiten sich in einer veränderten Politik niederschlagen können. Wie der nächste Abschnitt zeigen wird, ist es aus Sicht der Apologeten gerade das Ziel der Vergemeinschaftung, diesen Mechanismus zu durchbrechen. Doch damit trägt die EU zur wachsenden Verdrossenheit der „unzufriedenen Demokraten" bei, die zwar die Demokratie als Regierungsform befürworten, aber von der tatsächlichen Funktionsweise enttäuscht sind (Klingemann 2000, S. 267).

\subsection{Die Apologeten}

Aus Sicht der Apologeten resultiert Unzufriedenheit nicht aus einem Mangel an Beteiligung, sondern aus der Unfähigkeit der Politik, grundlegende Bedürfnisse der Wähler zu befriedigen. Hohe Arbeitslosigkeit, wachsende Staatsschulden und eine marode öffentliche Infrastruktur lassen die Bürger an der Problemlösungsfähigkeit von Regierungen zweifeln. Die Handlungsfähigkeit der Politik wieder herzustellen, ist der Zweck europäischer Integration. Die selektive Kompetenzübertragung auf EU-Institutionen soll das Politikversagen der Mitgliedstaaten ausgleichen.

Dabei prüfen nationale Regierungen genau, in welchen Bereichen sie Souveränitätsverluste akzeptieren. In umstrittenen Politikfeldern herrscht noch immer die Norm der Einstimmigkeit. Besonders groß ist die Autonomie der EU-Institutionen dagegen in Bereichen, in denen Kooperationsgewinne durch opportunistisches Verhalten gefährdet werden und in denen die technische Komplexität hoch ist. Kurzfristiges Denken und Trittbrettfahrerverhalten mögen individuell rational sein, führen aber zu kollektiv unerwünschten Ergebnissen. Im Bewusstsein der eigenen Schwäche binden sich Regierungen die Hände und übertragen Entscheidungs- und Überwachungskompetenzen an europäische Regulierungsbehörden, an die Europäische Kommission und den Europäischen Gerichtshof (EuGH). Die europäische Integration verdrängt den Nationalstaat nicht, sondern gewährleistet dessen Handlungsfähigkeit (Milward 2000, S. 44). Wo es jedoch vornehmlich um eine effektive Umsetzung unstrittiger Ziele geht, ist eine Politisierung der Entscheidungen kontraproduktiv. Eine Bürokratie, die sachlich und zielgerichtet arbeitet, muss vom politischen Tagesgeschäft abgeschottet werden. Wie die nationale Politik wäre eine politisierte EU lähmendem Parteienstreit und Interessengruppen ausgesetzt. Eine Demokratisierung der EU ist aus dieser Sicht zwar möglich, aber nicht erstrebenswert.

Moravcsiks (2002) Ausgangspunkt ist die Feststellung, dass der autonome Einfluss der EU übertrieben wird. Anknüpfend an die intergouvernementale Lesart der Integration bestreitet er den Kontrollverlust der Mitgliedstaaten und damit das EU-Demokratiedefizit. Im Zentrum europäischer Politik steht weiterhin der Ministerrat, in dem nationale Regierungen vertreten sind. Wo Kommission oder EuGH unabhängig entscheiden, liegt eine bewusste Strategie der Regierungen zugrunde, 
Transaktionskosten zu reduzieren. Zudem folgt die Delegation von Entscheidungsbefugnissen in der EU dem gleichen Muster wie im Nationalstaat. Auch in den Mitgliedstaaten werden die Verantwortung für die Geldpolitik, die Arzneimittelzulassung oder die Regulierung des Telefonmarkts, wie auch die Rechtsprechung an Experten übertragen, die nicht unmittelbar aus Wahlen hervorgehen. EU-Institutionen erfüllen also nur jene wohlfahrtsfördernden Aufgaben, die auch innenpolitisch an unabhängige Behörden übertragen werden (Moravcsik 2002, S. 613). Regierungen dokumentieren durch die Delegation von Befugnissen die Glaubwürdigkeit ihrer wirtschaftspolitischen Absichten (Moravcsik 1998, S. 73-77). Spezialisierte Behörden verwirklichen gemeinsam beschlossene Ziele und erhöhen die Leistungsfähigkeit des politischen Systems. Die Entscheidungen der EU sind nicht nur sachlich gerechtfertigt, sondern auch hinreichend legitimiert. Ein Demokratiedefizit der EU besteht nur, falls ein unrealistisches Ideal als Bewertungsmaßstab herangezogen wird, das selbst die Mitgliedstaaten nicht erfüllen (Moravcsik 2004, S. 337).

Auch für Majone (2000) wiegen die Vorteile der Delegation mögliche Nachteile auf. Der Transfer von Kompetenzen ist gerechtfertigt, wo sachgerechte Entscheidungen durch Regulierungsbehörden ein mögliches Marktversagen ausgleichen. Delegation dient der Verwirklichung von Pareto-Verbesserungen (Majone 1994, S. 81). In Abgrenzung zur Kritik am Demokratiedefizit verteidigt Majone technokratische Entscheidungen. Nicht direkt legitimierte Institutionen rechtfertigen sich über den Wohlfahrtsgewinn, der mit ihren Entscheidungen einhergeht. Da Pareto-Verbesserungen per definitionem niemanden schlechter stellen, gibt es keinen Grund für eine Politisierung der Beschlussfassung. Im Gegenteil, wenn die Ursache für die Delegation von Aufgaben an unabhängige Behörden in den Nachteilen politischer Verhandlungen liegt, wäre es schädlich, dem vermeintlichen Demokratiedefizit durch die Aufwertung der Politik zu begegnen. Genau diesen Trend beobachtet Majone jedoch in der EU. Die politische Kontrolle der Kommission durch nationale Regierungen, die die Kommissare entsenden, und die Aufwertung des Europäischen Parlaments drängen den neutralen Sachverstand zurück und gefährden jene Glaubwürdigkeit, die durch Delegation erzielt werden soll:

„[...] the progressive politicization and parliamentarization of the Commission raise again the issue of credibility, this time at the European level. A less technocratic, more political Commission may enjoy greater democratic legitimacy, but eventually it will have to face the same commitment problem of all democratic governments" (Majone 2000, S. 289).

Für die Argumentation beider Autoren ist die Annahme wichtig, dass Delegation die Handlungsfähigkeit der Politik verbessert. Sie verweisen auf die legitimierende Wirkung guten Regierens (Output-Legimität). Bei Input-Legitimität geht es darum, politische Entscheidungen möglichst unverfälscht aus dem Willen des Souveräns abzuleiten. Aus der Output-Perspektive sollen dagegen die Interessen der Wähler möglichst wirksam gefördert werden (Scharpf 2004, S. 5). Die verbesserte Leistungsfähigkeit des politischen Systems begründet sowohl die Delegation von Entschei- 
dungsbefugnissen als auch Mehrheitsabstimmungen im Ministerrat und das Initiativrecht der Kommission. Aufgrund der Interessenvielfalt der Mitgliedstaaten müssen Verfahren gefunden werden, die Entscheidungen erleichtern. Eine verbesserte Effizienz und Effektivität des politischen Systems wiegen den Mangel an Beteiligung, demokratischem Streit und Transparenz auf.

Die Apologeten nehmen demnach eine den Optimisten entgegengesetzte Position ein. Während diese die EU-Politik über mehr Bürgerbeteiligung legitimieren wollen, kann die Union für jene nur als Problemlöser legitim sein. Nicht in einer zu geringen Bürgerbeteiligung liegt das Problem der Mitgliedstaaten, sondern in vielfältigen Blockaden. Die nationale Politik ist im Konsenszwang gefangen, unfähig, Reformen mit der nötigen Geschwindigkeit und Konsequenz umzusetzen. Eine gewählte Regierung sollte jedoch für die Dauer einer Legislaturperiode über die Macht verfügen, das eigene Programm umzusetzen. Das erfordert Unabhängigkeit von Gruppen, die ihre Partikularinteressen über das Gemeinwohl stellen. Da in vielen Mitgliedstaaten die Regierungen von "Sonderinteressengruppen“ abhängig sind, bietet erst die vermeintliche Abgabe von Souveränität die Chance, sie tatsächlich auszuüben. Hier gleichen die Argumente der Apologeten Olsons Niedergangsthese. Auch er sah in der "hoheitlichen Integration“ einen Weg, die Unabhängigkeit der Politik von nationalen Verteilungskoalitionen zu erhöhen (Olson 1991, S. 168, Kapitel 5). Die Europäische Union stärkt die Exekutive und befreit sie aus korporatistischen Verhandlungszwängen (Moravcsik 1997; Mair 2005, S. 22). ${ }^{6}$

Alle Versuche, die EU zu politisieren, verkennen den Zweck der Integration. Die EU tritt an die Stelle der Mitgliedstaaten, wo diese ihre Aufgaben nicht erfüllen können. Politische Entscheidungen werden dem Parteienstreit entzogen, um die Qualität des Regierens zu verbessern. Das Leitbild der Apologeten ist eine aufgeklärte Bürokratie, die im Interesse der Bevölkerung entscheidet. Da „regulative Politik“ unkontrovers und verteilungsneutral ist, bedarf sie keiner zusätzlichen Legitimierung. Im Gegenteil, eine Politisierung der Europäischen Union liefe ihrem Aufgabenprofil zuwider.

\subsection{Die Fatalisten}

Die Autoren des vorherigen Abschnitts begründen, weshalb institutionelle Reformen zur Demokratisierung der EU ausbleiben, obwohl sie prinzipiell möglich wären. Die Fatalisten teilen die Einschätzung, dass die Europäische Union keine Mehrheitsdemokratie werden kann. Aus ihrer Sicht gefährden demokratische Reformen nicht bloß die Problemlösungsfähigkeit des politischen Systems, sondern im gravie-

6 Obwohl Olsons Theorie der Sonderinteressengruppen allgemein formuliert ist, wird an vielen Stellen seines Buches deutlich, dass er vor allem die Gewerkschaften für wirtschaftliche Stagnation verantwortlich macht (z.B. Olson 1991, S. 142). Auch Moravcsik (2002, S. 618) sieht in der EU eine willkommene Korrektur übertriebener Sozialstaatlichkeit. 
rendsten Fall den Fortgang der Integration. Die Europäische Union braucht vertrauensbildende Maßnahmen und Mechanismen des Ausgleichs. Von Beginn an war die europäische Integration ein Elitenprojekt, das auf hinter verschlossenen Türen ausgehandelte Kompromisse angewiesen war. Abgeschottet von populären Erwartungen entstand eine Form diffuser Reziprozität, die politischen Tausch erleichterte. Regierungsvertreter, die bei jedem Zugeständnis die Kritik der Opposition und den Zorn der Bevölkerung befürchten, können nicht effektiv verhandeln. Die Erfolge der europäischen Integration beruhen darauf, dass sie hinter dem Rücken der Bevölkerung von den nationalen Eliten ausgehandelt wurden. Eine transparente und demokratische EU birgt ein Konfliktpotenzial, das die Integration selbst gefährdet.

In der EU wird die Macht geteilt. Verhandlungen, nicht Hierarchie sind der typische Koordinierungsmechanismus. In einem sprachlich und kulturell zerklüfteten, von unterschiedlichen historischen Erfahrungen geprägten Gemeinwesen muss die Zentralisierung der Macht für Minderheiten bedrohlich wirken. Die Entscheidungen der Mehrheit erscheinen den numerisch Unterlegenen nur anerkennungswürdig, falls garantiert ist, dass ihre Belange berücksichtigt werden. Folglich waren bis zur Einheitlichen Europäischen Akte (1987) alle EU-Entscheidungen zumindest informell dem Einstimmigkeitsprinzip unterworfen. Jedes Mitgliedsland wurde gleich behandelt - unabhängig davon, wie viele Menschen die Regierung repräsentierte. Mit dem Übergang zu Mehrheitsabstimmungen ist dieses Prinzip gelockert worden. Doch selbst bei qualifizierter Mehrheit müssen fast drei Viertel der Stimmen im Ministerrat erzielt werden. Zudem betont das Subsidiaritätsprinzip die Autonomie der Mitgliedstaaten, und die Vergabe von wichtigen Ämtern folgt dem Proporz. Die Europäische Union wird daher als Konkordanzsystem (Puchala 1972) oder konsoziativer Staat (Schmidt 2000) bezeichnet. Aus dieser Charakterisierung ergibt sich, dass nicht alle Wege für eine Demokratisierung der EU offen stehen:

„Consociational theorists seek to show how, in all successful consociational democracies, normal tradition political fora were bypassed, and substituted by fora in which the leaders of all social segments participated, and compacts were arrived at, disregarding the principle of majority rule and using instead consensual politics" (Weiler/Haltern/Mayer 1995, S. 29).

Die Prinzipien eines konsoziativen Staats sind nicht mit dem Anliegen der Optimisten vereinbar, die EU für eine breite Bürgerbeteiligung zu öffnen und in eine parlamentarische Demokratie weiterzuentwickeln. Drei Gründe sind dafür verantwortlich. Erstens setzen erfolgreiche Verhandlungen voraus, dass die Ergebnisse nicht wieder aufgeschnürt werden. Die Verhandlungspartner müssen sich darauf verlassen können, dass die getroffenen Vereinbarungen eingehalten werden. Nationale Parlamente stimmen daher nur über das Gesamtpaket ab. Selbst Änderungen im Detail gefährden den mühsam ausgehandelten Kompromiss. Das bedeutet zweitens, dass eine Kontrolle der Verhandlungsführer weder vorab noch im Nachhinein möglich ist. Mechanismen der Überwachung durch die Opposition oder die Medien greifen nicht, wenn Entscheidungen in Elitenkartellen getroffen werden und unveränder- 
lich erscheinen. Drittens, das für eine erfolgreiche Konfliktbearbeitung nötige Vertrauen entsteht, weil sich die Verhandlungspartner kennen und auf erfolgreiche Kompromisse zurückblicken können. Eine Öffnung des Verhandlungszirkels gefährdet dieses Vertrauen. Das Erfolgsgeheimnis der EU besteht also im Verzicht auf partizipatorische Entscheidungsverfahren.

Die Fatalisten argumentieren vor diesem Hintergrund, dass das Demokratiedefizit den Zusammenhalt der EU gewährleistet. Eine Demokratisierung der Union wäre nur um den Preis ihres Bestands zu haben und sei daher weder erstrebenswert noch möglich. Gabel (1998, S. 470-471) wendet sich ausdrücklich gegen das Anliegen der Optimisten, das Demokratiedefizit durch institutionelle Reformen zu beheben. Vor allem warnt er vor der Einführung mehrheitsdemokratischer Elemente. Gerade Faktoren, die als Ursache des Demokratiedefizits angegeben werden - die fehlende Kontrolle der Eliten, Intransparenz und mangelnde Teilhabechancen -, begründen den Erfolg der europäischen Integration. Eine Abkehr von diesen Prinzipien könnte „potenziell katastrophale Folgen“ (Gabel 1998, S. 471) für die Stabilität der EU haben (siehe auch Chryssochoou 1994, S. 4). Solange die EU national segmentiert bleibt und die Herkunft andere Konfliktlinien überlagert, können politische Auseinandersetzungen nicht nach dem Mehrheitsprinzip erfolgen. Zu groß ist die Gefahr, dass strukturelle Minderheiten entstehen, die sich dauerhaft im Nachteil sehen. Ohne eine „belastungsfähige Kommunikationsgemeinschaft" und "gemeinschaftsbezogene Loyalitäten“ ist eine Demokratisierung der EU nicht möglich (Schmidt 2000, S. 48-49).

\section{Wie demokratisch und wie demokratiefähig ist die Europäische Union?}

Nach der vorangegangenen Darstellung der vier Positionen zum EU-Demokratiedefizit soll im Folgenden gezeigt werden, welche Beschreibung der Europäischen Union empirisch angemessen erscheint.

Mit Grande (1996) lässt sich die „Standardvariante“ der Kritik am EU-Demokratiedefizit, die an institutionellen Funktionsmängeln des politischen Systems ansetzt, von der „substanziellen“ oder strukturellen Variante unterscheiden, die die Demokratisierbarkeit grundsätzlich bestreitet. Während die Standardvariante eine weitere Parlamentarisierung und die Aufwertung der EP-Wahlen als angemessene Antwort auf das Demokratiedefizit betrachtet, erscheint die EU aus Sicht der strukturellen Kritik schlicht als nicht "demokratiefähig“" (Kielmansegg 2003, S. 57-61). Optimisten und Apologeten sind der ersten Variante, Pessimisten und Fatalisten der zweiten zuzuordnen. Die vier Positionen unterscheiden sich zudem hinsichtlich ihres Demokratieverständnisses. Optimisten und Pessimisten konzentrieren ihre Argumente auf Input-orientierte Legitimation, während Apologeten und Fatalisten die Output-orientierte Legitimation in den Vordergrund stellen. Dieser Abschnitt konzentriert sich darauf, ob und welche Art der Demokratisierung der EU möglich ist. 
Abbildung 2: Zufriedenheit mit der Demokratie

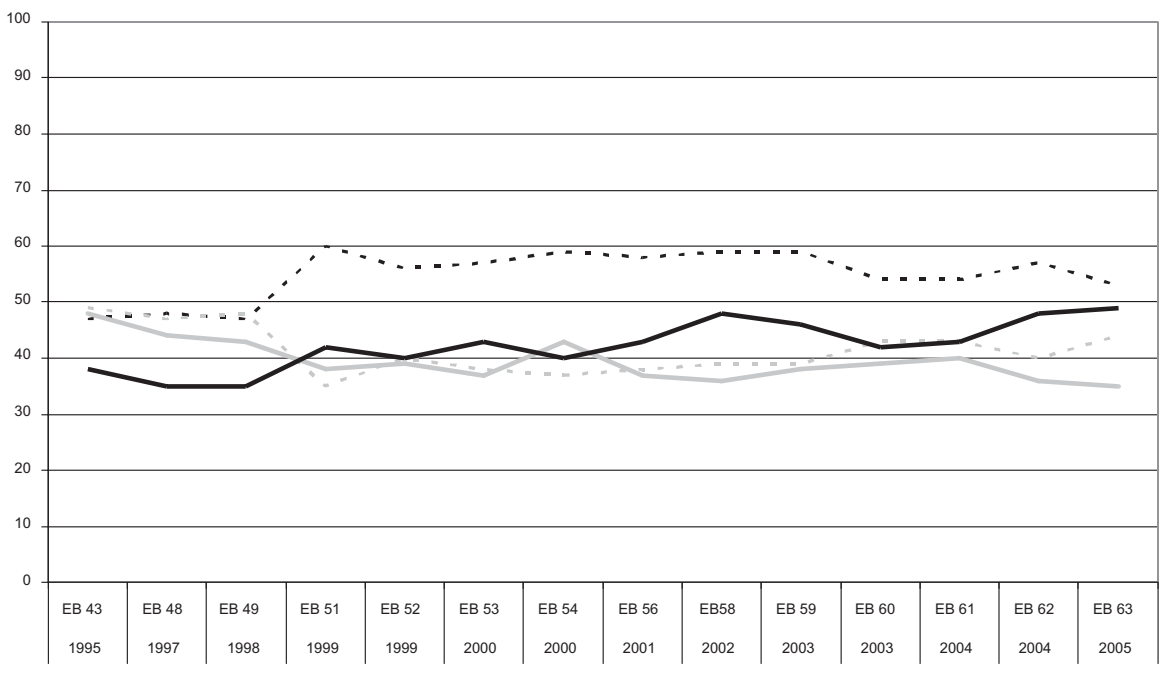

- - - Nat. zufrieden

Nat. unzurfieden

EU unzurrieden

EU zurrieden

Daten: Eurobarometer 63, September 2005, S. 21-24.

Die Bewertung ihrer Wünschbarkeit entzieht sich dem direkten empirischen $\mathrm{Zu}$ griff. Dennoch informiert der Blick auf den Ist-Zustand der EU-Demokratie über mögliche Reformperspektiven. Für Apologeten und Fatalisten ist insbesondere die Problemlösungsfähigkeit der EU wichtig. Nur wenn sich zeigen lässt, dass die Unionsbürger die EU-Politik als wohlfahrtsteigernd anerkennen, erscheint der Fokus auf Output-Legimität plausibel. Auf der anderen Seite steht für die Optimisten die Frage im Vordergrund, wie sich vergangene Reformen auf die Partizipation der Bürger ausgewirkt haben.

Ein erster Schritt betrachtet, wie es aus Sicht der Bevölkerung um die nationale und die EU-Demokratie bestellt ist. Seit 1993 wird in Eurobarometer-Umfragen regelmäßig nach der Zufriedenheit mit der Demokratie in der EU gefragt. Nachdem zu Beginn eine relative Mehrheit der Befragten angab, unzufrieden zu sein, antworteten zuletzt 49 Prozent, sie seien zufrieden, während 35 Prozent weiterhin Unzufriedenheit mit der Art und Weise äußerten, wie die Demokratie in der Europäischen Union funktioniert (Abbildung 2). Diese Werte liegen nicht gravierend unterhalb der durchschnittlichen Zustimmung zur Demokratie in den Mitgliedstaaten. ${ }^{7}$ Doch die Durchschnittswerte verdecken große Unterschiede zwischen den Ländern.

7 Doch hinter dieser Annäherung verbergen sich einerseits die extrem niedrige Zufriedenheit mit der nationalen Demokratie in den neuen Mitgliedstaaten (NMS 33\%; EU-15 57\%) und andererseits ihre relativ hohe Zustimmung zur EU-Demokratie (NMS 53\%; EU-15 48\%). 
Abbildung 3: Wahlbeteiligung in den EU-25 Staaten

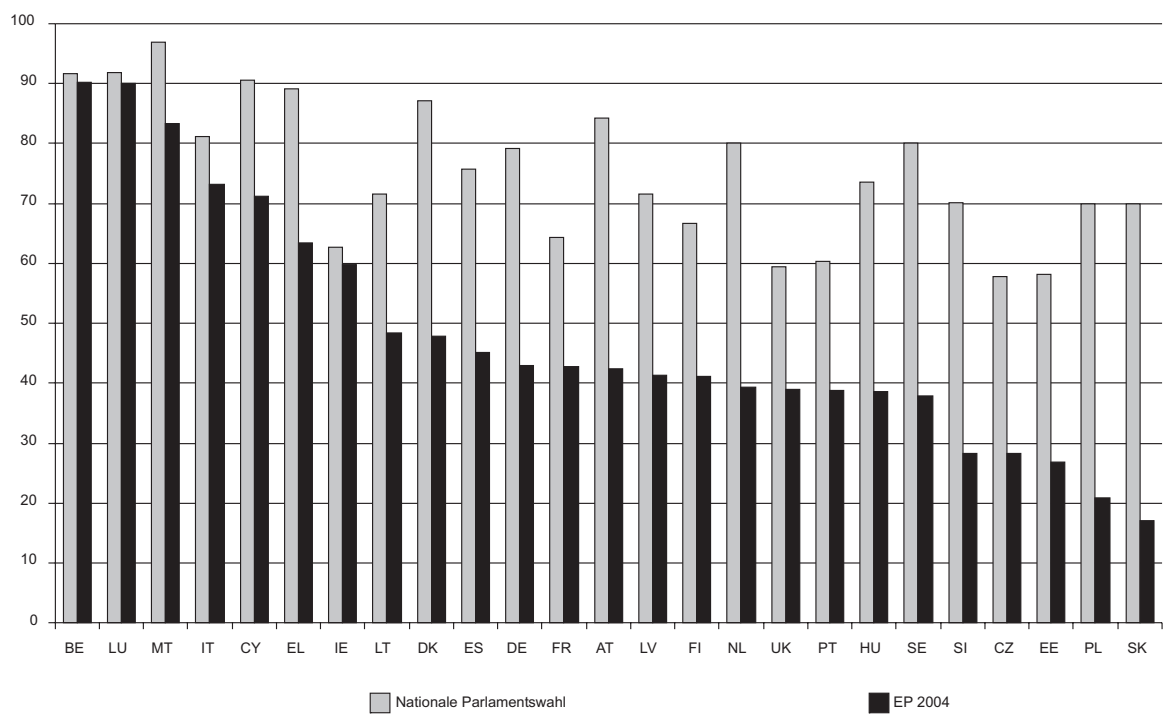

Daten: Europaparlament; European Journal of Political Research; Rose/Munro (2003).

In Dänemark sind 92 Prozent der Befragten mit der nationalen Demokratie zufrieden, in Litauen nur 24 Prozent. Ähnlich verhält es sich mit der EU-Demokratie. In Luxemburg und Belgien äußern sich zwei Drittel zustimmend, während in den Niederlanden und dem Vereinigten Königreich nur vier von zehn Befragten zufrieden mit der EU-Demokratie sind. Für die Mitgliedstaaten zeigt sich ein starker und statistisch signifikanter Zusammenhang zwischen dem Wohlstandsniveau (gemessen am BIP pro Kopf relativ zum EU-Durchschnitt) und der Zufriedenheit der Bevölkerung mit der nationalen Demokratie $\left(r=.775^{* *} ; \mathrm{p}=.000 ; \mathrm{n}=25\right)$.

Werden die Befragten nicht allgemein nach der Zufriedenheit mit der Demokratie gefragt, sondern danach, ob ihre Stimme in der EU zählt, antwortet eine deutliche Mehrheit (55 Prozent) mit Nein, weniger als ein Drittel mit Ja (Eurobarometer 61, 2004). Über die Wahlen zum Europaparlament könnten die Bürger Einfluss ausüben, doch diese Möglichkeit nehmen sie nur sehr zurückhaltend in Anspruch. Seit der ersten Direktwahl des Europaparlaments ist die Wahlbeteiligung bei jeder Wahl zurückgegangen. Gaben 1979 noch mehr als 60 Prozent der Wähler ihre Stimme ab, waren es 2004 nur noch 45,5 Prozent. Abbildung 3 vergleicht die Wahlbeteiligung bei der Europawahl 2004 mit der vorherigen nationalen Parlamentswahl. Während die durchschnittliche Wahlbeteiligung in nationalen Wahlen bei 75,3 Prozent lag, nahmen nur 47,9 Prozent der Wahlberechtigten an den EP-Wahlen teil. Die Diskrepanz in der Wahlbeteiligung zwischen Haupt- und Nebenwahlen, die auch bei deutschen Landtagswahlen zu beobachten ist, spricht gegen eine generelle Wahlmüdig- 
Abbildung 4: Nationale versus proportionale Repräsentation

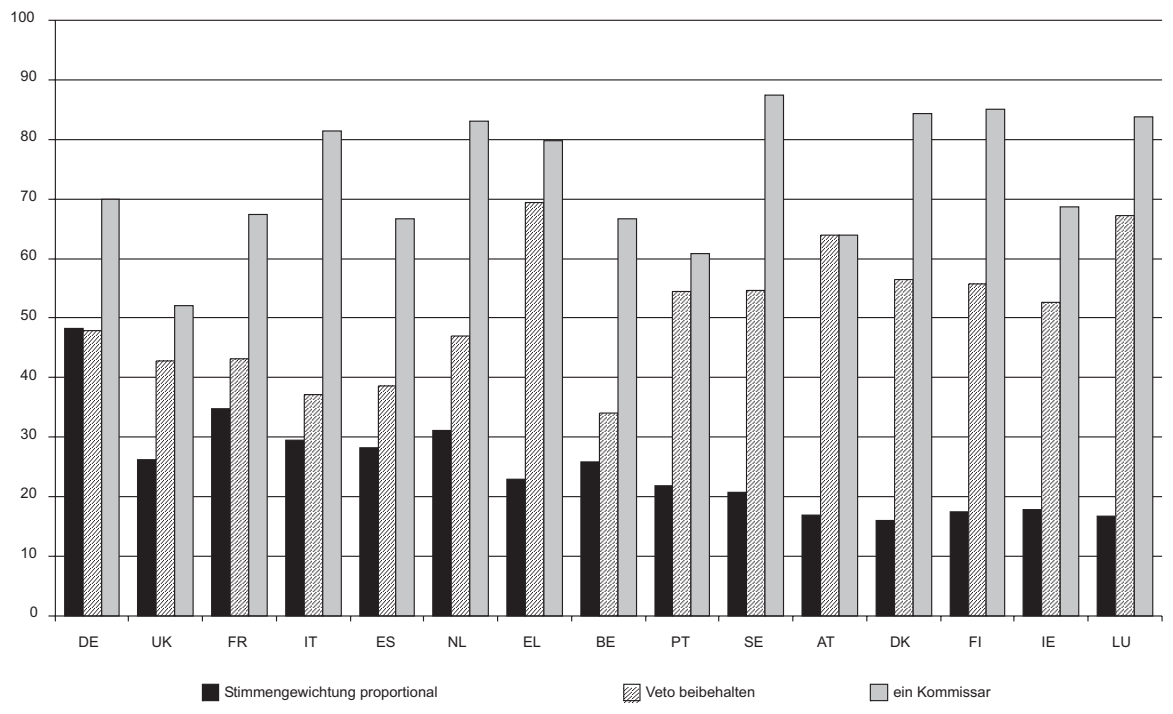

Daten: Eurobarometer 61, 2004, eigene Berechnung.

keit. Gleichwohl gefährdet eine hohe Wahlabstinenz die Legitimierungskraft des Europäischen Parlaments.

Nun könnte die Wahlbeteiligung niedrig sein, weil die Kompetenzen des Europaparlaments gering sind. Allerdings hat das EP mit jeder Vertragsänderung zusätzliche Befugnisse erhalten - und dennoch sank die Wahlbeteiligung. Im Widerspruch zur Reformstrategie der Optimisten lautet der Befund: Je wichtiger das Europaparlament wurde, desto niedriger war die Wahlbeteiligung. Eine weitere Parlamentarisierung der EU garantiert vor diesem Hintergrund keine Lösung für das Demokratiedefizit. Noch aus einem zweiten Grund ist die Verlagerung von mehr Kompetenzen auf das Europaparlament demokratietheoretisch angreifbar. Die Wähler bevölkerungsarmer Länder sind im EP deutlich überrepräsentiert, die großer Länder unterrepräsentiert. Ein deutscher Abgeordneter vertritt 829.000 Wähler, während ein Luxemburger lediglich 71.500 Wähler repräsentiert. Der Einfluss der Wählerstimmen ist in hohem Maß ungleich (Kneip/Petring 2006, S. 216-217). ${ }^{8}$ Würde man den Ministerrat in eine Zweite Kammer umwandeln, in der die Teilstaaten unabhängig von ihrer Größe gleich behandelt werden, müsste zuvor die Repräsentativität des EP gestärkt werden - doch dies entspricht nicht der Vorstellung der meisten EU-Bürger.

8 Wollte man das Prinzip durchsetzen, dass jede Stimme gleich zählt, müsste Deutschland knapp 1150 Abgeordnete entsenden. Wollte man alternativ die aktuelle Größe von 732 Abgeordneten beibehalten, enthielte ein einzelner Wahlkreis mehr Stimmberechtigte als Luxemburg oder Malta Einwohner haben. 
Abbildung 5: Grad der Zugehörigkeit

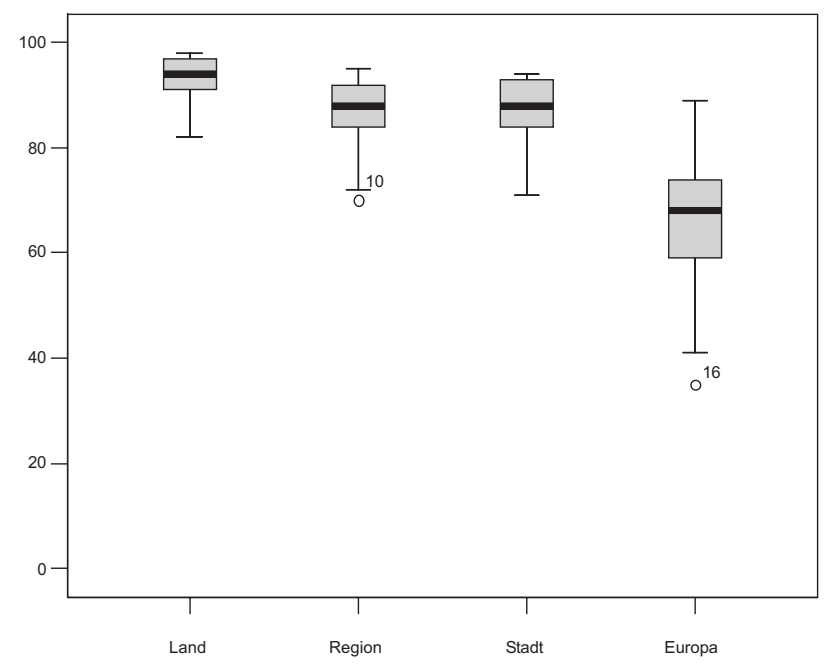

Quelle: Eurobarometer 62, eigene Berechnung aus Länderdurchschnitten.

Die Mehrheit der Bevölkerung sieht die Abkehr von einer intergouvernementalen EU skeptisch. In Abbildung 4 sind die 15 alten EU-Mitgliedstaaten nach ihrer Bevölkerungszahl von links nach rechts geordnet. Während sich in allen Mitgliedstaaten eine Mehrheit dafür ausspricht, dass die Anzahl der Kommissare nicht nach Ländergröße variiert, sondern jedes Mitgliedsland einen Kommissar stellen soll, zeigt sich ein klarer Zusammenhang zwischen der Ländergröße und der Anzahl derjenigen, die eine proportionale Stimmengewichtung im Ministerrat bevorzugen. Je größer ein Land, desto weniger Menschen stimmen der Aussage zu, dass jeder Mitgliedstaat, unabhängig von seiner Größe, nur eine Stimme im Ministerrat haben sollte $\left(\mathrm{r}=-.83^{* *} ; \mathrm{p}=.000 ; \mathrm{n}=15\right)$. Die Befragten in kleineren Ländern wollen dagegen den nationalen Einfluss mit einem eigenen Kommissar, durch das Beibehalten nationaler Vetorechte und durch das Gleichheitsprinzip der Mitgliedstaaten wahren. Das nationale Interesse dominiert die Sicht auf die Europäische Union. Solange dies so ist, bleibt der Weg zur repräsentativen Demokratie versperrt.

Die Pessimisten gehen davon aus, dass durch Entgrenzung Solidarbeziehungen im Nationalstaat unterminiert werden. Für die Zukunft der Demokratie in der EU ist entscheidend, ob eine grenzüberschreitende Loyalität neben die nationale tritt. Die Demokratiefähigkeit der EU wird mit dem Argument bezweifelt, dass es keine „politisch belastbare Identität der Europäer als Europäer“ gibt (Kielmansegg 2003, S. 57). Auch diese Frage lässt sich prinzipiell empirisch klären, auch wenn die vorliegenden Daten nicht viel mehr als ein Fingerzeig sind. Abbildung 5 gibt Auskunft darüber, wie viele Bürger angaben, sich ihrem Land, ihrer Region, der Stadt oder Europa verbunden zu fühlen. Die Boxplot-Grafik bildet den Medianwert ab und vermit- 
telt, wie stark die Werte streuen. Dabei wird deutlich, dass die Verbundenheit mit der EU nicht nur deutlich niedriger als die mit Land, Region oder Stadt ist, sondern dass auch die nationalen Durchschnittswerte stark voneinander abweichen. Die Standardabweichung ist für die Europawerte $(13,8)$ dreimal so hoch wie die für die nationale Zugehörigkeit $(4,4)$.

Während also die Zugehörigkeit zum eigenen Land besonders stark empfunden wird, gibt es überlappende Identitäten. Doch wie sind sie gewichtet? 2004 gaben 41 Prozent der Befragten an, sich nur ihrer Nation zugehörig zu fühlen. Weitere 47 Prozent sahen sich zwar auch als Europäer, aber erst an zweiter Stelle. Sieben Prozent begriffen sich zuerst als Europäer, dann als Spanier, Schweden oder Slowenen. Drei Prozent fühlten sich nur als Europäer. Daraus lässt sich schließen, dass bei der überwiegenden Mehrheit der Unionsbürger die nationale Identität Vorrang vor der europäischen hat. Dies muss nicht dauerhaft so bleiben, aber jede Veränderung wird sich langsam vollziehen. Das Argument der Pessimisten beruht nicht auf der Annahme, dass Nationen natürlich gegeben und Umverteilungsgemeinschaften unveränderlich sind (Bartolini 2005, S. 409). Dennoch bildet sich eine belastbare Identität - auch als Verfassungspatriotismus - nur über einen langen Zeitraum und innerhalb definierter Grenzen heraus.

Uneinheitlich sind die Befunde hinsichtlich der Frage, inwieweit die Bürger eine redistributive EU-Politik als Ausdruck einer Solidarität jenseits des Nationalstaats befürworten. Im Eurobarometer 60 wurde nach der Aufgabenteilung zwischen den Mitgliedstaaten und der EU gefragt. Mehr als zwei Drittel sehen den Kampf gegen internationalen Terrorismus, Menschenhandel, Ausbeutung, organisiertes Verbrechen und Drogen primär als Aufgabe der EU an. Auch eine gemeinsame Außenpolitik und humanitäre Hilfe werden stark unterstützt. Darüber hinaus befürworten fast 60 Prozent EU-Kompetenzen im Kampf gegen Armut sowie soziale Ausgrenzung und zur Unterstützung wirtschaftlich schwacher Regionen. In den klassischen Feldern der Arbeitsmarktpolitik sowie im Gesundheits- und Sozialwesen votierten dagegen 51 bzw. 67 der Befragten für nationale Entscheidungen (Eurobarometer 60, 2003, S. 70-71). Hier scheint die Bereitschaft begrenzt, Kompetenzen und Ressourcen zu transferieren. Zudem sehen diejenigen eine Vergemeinschaftung der Sozialpolitik am skeptischsten, die befürchten, dass die EU zu einem Rückbau von Sozialleistungen führt (Mau 2003, S. 316). Je stärker der Sozialstaat unter Druck gerät, desto entschiedener lehnen die Betroffenen die Europäisierung der sozialen Sicherung ab.

Betrachtet man die Bereitschaft zu grenzüberschreitender Solidarität, fallen ausgeprägte Unterschiede zwischen Ländern ins Auge. Während in Luxemburg, Irland und den Niederlanden mehr als 60 Prozent äußern, sie würden höhere Steuern zahlen, um einem anderen EU-Staat zu helfen, tun dies in Belgien, Portugal und Finnland weniger als ein Drittel der Befragten. In den restlichen Ländern halten sich Befürworter und Gegner etwa die Waage. Aufschlussreich ist dabei, welche Konfliktlinien diese beiden Gruppen trennen. Tabelle 1 informiert über die Faktoren, die die 
Tabelle 1: Grenzüberschreitende Solidarität

\begin{tabular}{|c|c|c|c|c|c|c|c|c|c|}
\hline & & $\begin{array}{r}E U-15 \\
\mathrm{~N}=8046\end{array}$ & $\begin{array}{r}L \\
\mathrm{~N}=211\end{array}$ & $\begin{array}{r}I R L \\
\mathrm{~N}=395\end{array}$ & $\begin{array}{r}N L \\
\mathrm{~N}=758\end{array}$ & $\begin{array}{r}E S \\
\mathrm{~N}=670\end{array}$ & $\begin{array}{r}D \\
\mathrm{~N}=868\end{array}$ & $\begin{array}{r}D K \\
\mathrm{~N}=695\end{array}$ & $\begin{array}{r}A \\
\mathrm{~N}=390\end{array}$ \\
\hline & & $\begin{array}{r}\text { Exp (B) } \\
\text { (Wald) }\end{array}$ & $\begin{array}{r}\text { Exp (B) } \\
\text { (Wald) }\end{array}$ & $\begin{array}{r}\text { Exp (B) } \\
\text { (Wald) }\end{array}$ & $\begin{array}{r}\text { Exp (B) } \\
\text { (Wald) }\end{array}$ & $\begin{array}{r}\text { Exp (B) } \\
\text { (Wald) }\end{array}$ & $\begin{array}{l}\operatorname{Exp}(B) \\
\text { (Wald) }\end{array}$ & $\begin{array}{r}\operatorname{Exp}(B) \\
\text { (Wald) }\end{array}$ & $\begin{array}{r}\text { Exp (B) } \\
\text { (Wald) }\end{array}$ \\
\hline Alter & & $\begin{array}{r}1^{* *} \\
(11,85)\end{array}$ & $\begin{array}{r}1 \\
(0,02)\end{array}$ & $\begin{array}{r}0,99 \\
(1,79)\end{array}$ & $\begin{array}{r}1 \\
(0,97)\end{array}$ & $\begin{array}{r}1 \\
(0,01)\end{array}$ & $\begin{array}{r}1,02^{* *} \\
(17,26)\end{array}$ & $\begin{array}{r}1,02^{* *} \\
(10,77)\end{array}$ & $\begin{array}{r}1,02^{*} \\
(6,17)\end{array}$ \\
\hline $\begin{array}{l}\text { Geschlecht } \\
\text { (weiblich) }\end{array}$ & & $\begin{array}{r}0,96 \\
(0,89)\end{array}$ & $\begin{array}{r}0,75 \\
(0,35)\end{array}$ & $\begin{array}{r}1,13 \\
(0,27)\end{array}$ & $\begin{array}{l}0,62^{* *} \\
(7,96)\end{array}$ & $\begin{array}{r}0,88 \\
(0,02)\end{array}$ & $\begin{array}{r}0,88 \\
(0,74)\end{array}$ & $\begin{array}{r}0,85^{*} \\
(0,97)\end{array}$ & $\begin{array}{r}1,48 \\
(2,55)\end{array}$ \\
\hline soziale Klasse & & $\begin{array}{l}1,14^{* *} \\
(28,8)\end{array}$ & $\begin{array}{r}1,28 \\
(1,76)\end{array}$ & $\begin{array}{r}0,81 \\
(3,57)\end{array}$ & $\begin{array}{r}1,02 \\
(0,05)\end{array}$ & $\begin{array}{l}1,27^{* *} \\
(7,53)\end{array}$ & $\begin{array}{l}1,19^{*} \\
(4,49)\end{array}$ & $\begin{array}{r}1,03 \\
(0,15)\end{array}$ & $\begin{array}{r}1,24 \\
(2,46)\end{array}$ \\
\hline \multirow{2}{*}{ Bildung } & mittel & $\begin{array}{r}1,18^{*} \\
(5,98)\end{array}$ & $\begin{array}{l}0,49 \\
(2,2)\end{array}$ & $\begin{array}{r}0,79 \\
(0,59)\end{array}$ & $\begin{array}{r}1,06 \\
(0,03)\end{array}$ & $\begin{array}{r}1,19 \\
(0,56)\end{array}$ & $\begin{array}{l}1,85^{* *} \\
(7,38)\end{array}$ & $\begin{array}{r}0,99 \\
(0,00)\end{array}$ & $\begin{array}{r}0,95 \\
(0,03)\end{array}$ \\
\hline & hoch & $\begin{array}{l}1,63^{* *} \\
(44,3)\end{array}$ & $\begin{array}{r}1,4 \\
(0,37)\end{array}$ & $\begin{array}{r}1,86 \\
(2,73)\end{array}$ & $\begin{array}{r}1,37 \\
(0,83)\end{array}$ & $\begin{array}{r}1,11 \\
(0,19)\end{array}$ & $\begin{array}{r}2,64^{* *} \\
(15,21)\end{array}$ & $\begin{array}{r}1,86 \\
(2,38)\end{array}$ & $\begin{array}{c}2,81^{* *} \\
(7,32)\end{array}$ \\
\hline $\begin{array}{l}\text { ideolog. } \\
\text { Einordnung }\end{array}$ & & $\begin{array}{l}0,91^{* *} \\
(79,2)\end{array}$ & $\begin{array}{l}0,85 \\
(3,3)\end{array}$ & $\begin{array}{r}0,93 \\
(1,77)\end{array}$ & $\begin{array}{r}0,87^{* *} \\
(13,89)\end{array}$ & $\begin{array}{r}0,95 \\
(3,05)\end{array}$ & $\begin{array}{r}0,9^{* *} \\
(7,35)\end{array}$ & $\begin{array}{r}0,92^{*} \\
(5,16)\end{array}$ & $\begin{array}{r}0,88 \\
(3,11)\end{array}$ \\
\hline $\begin{array}{l}\text { EU-Unter- } \\
\text { stützung }\end{array}$ & & $\begin{array}{r}1,18^{* *} \\
(356,04\end{array}$ & $\begin{array}{r}1,26^{* *} \\
(11,92)\end{array}$ & $\begin{array}{r}1,18^{* *} \\
(12,95)\end{array}$ & $\begin{array}{r}1,28^{* *} \\
(38,59)\end{array}$ & $\begin{array}{r}1,18^{* *} \\
(28,05)\end{array}$ & $\begin{array}{c}1,29^{* *} \\
(60,21)\end{array}$ & $\begin{array}{r}1,22^{* *} \\
(38,04)\end{array}$ & $\begin{array}{r}1,44^{* *} \\
(49,16)\end{array}$ \\
\hline \multirow{2}{*}{$\begin{array}{l}\text { politisches } \\
\text { Interesse }\end{array}$} & mittel & $\begin{array}{c}1,51^{* *} \\
(54,41)\end{array}$ & $\begin{array}{r}0,72 \\
(0,69)\end{array}$ & $\begin{array}{r}2,38^{* *} \\
(9,22)\end{array}$ & $\begin{array}{r}1,36 \\
(2,88)\end{array}$ & $\begin{array}{l}1,18 \\
(0,6)\end{array}$ & $\begin{array}{r}1,59 * \\
(5,52)\end{array}$ & $\begin{array}{r}1,41 \\
(2,36)\end{array}$ & $\begin{array}{r}1,31 \\
(0,83)\end{array}$ \\
\hline & hoch & $\begin{array}{r}2,02^{* *} \\
(129,72\end{array}$ & $\begin{array}{r}1,39 \\
(0,48)\end{array}$ & $\begin{array}{r}2,63^{* *} \\
(10,2)\end{array}$ & $\begin{array}{l}2,09^{* *} \\
(7,64)\end{array}$ & $\begin{array}{r}1,1 \\
(0,2)\end{array}$ & $\begin{array}{c}2,34^{* *} \\
(15,59)\end{array}$ & $\begin{array}{l}1,81^{* *} \\
(7,49)\end{array}$ & $\begin{array}{l}2,18^{*} \\
(6,17)\end{array}$ \\
\hline Konstante & & $\begin{array}{r}0,34^{* *} \\
(89,07)\end{array}$ & $\begin{array}{r}0,82 \\
(0,06)\end{array}$ & $\begin{array}{c}3,63^{*} \\
(6,11)\end{array}$ & $\begin{array}{r}0,61 \\
(1,09)\end{array}$ & $\begin{array}{r}0,46^{*} \\
(3,9)\end{array}$ & $\begin{array}{r}0,08^{* *} \\
(42,87)\end{array}$ & $\begin{array}{r}0,19^{* *} \\
(16,05)\end{array}$ & $\begin{array}{r}0,07^{* *} \\
(15,45)\end{array}$ \\
\hline \multirow[t]{3}{*}{ Nagelkerke $R^{2}$} & & 0,137 & 0,208 & 0,142 & 0,166 & 0,098 & 0,242 & 0,142 & 0,37 \\
\hline & & $\begin{array}{r}F \\
\mathrm{~N}=621\end{array}$ & $\begin{array}{r}U K \\
\mathrm{~N}=735\end{array}$ & $\begin{array}{r}E L \\
\mathrm{~N}=397\end{array}$ & $\begin{array}{r}S \\
\mathrm{~N}=323\end{array}$ & $\mathrm{~N}=1086$ & $\begin{array}{r}B \\
\mathrm{~N}=294\end{array}$ & $\begin{array}{r}P \\
\mathrm{~N}=298\end{array}$ & $\begin{array}{r}F I N \\
\mathrm{~N}=305\end{array}$ \\
\hline & & $\begin{array}{r}\text { Exp (B) } \\
\text { (Wald) }\end{array}$ & $\begin{array}{r}\text { Exp (B) } \\
\text { (Wald) }\end{array}$ & $\begin{array}{r}\operatorname{Exp}(B) \\
\text { (Wald) }\end{array}$ & $\begin{array}{r}\operatorname{Exp}(B) \\
\text { (Wald) }\end{array}$ & $\begin{array}{r}\text { Exp (B) } \\
\text { (Wald) }\end{array}$ & $\begin{array}{l}\operatorname{Exp}(B) \\
\text { (Wald) }\end{array}$ & $\begin{array}{r}\operatorname{Exp}(B) \\
\text { (Wald) }\end{array}$ & $\begin{array}{r}\text { Exp (B) } \\
\text { (Wald) }\end{array}$ \\
\hline Alter & & $\begin{array}{r}1 \\
(0,15)\end{array}$ & $\begin{array}{r}1 \\
(0,01)\end{array}$ & $\begin{array}{r}1 \\
(0,25)\end{array}$ & $\begin{array}{l}1,02^{* *} \\
(7,17)\end{array}$ & $\begin{array}{r}1 \\
(0,96)\end{array}$ & $\begin{array}{r}0,99 \\
(0,45)\end{array}$ & $\begin{array}{r}0,99 \\
(0,52)\end{array}$ & $\begin{array}{r}1,01 \\
(0,69)\end{array}$ \\
\hline $\begin{array}{l}\text { Geschlecht } \\
\text { (weiblich) }\end{array}$ & & $\begin{array}{r}0,78 \\
(1,96)\end{array}$ & $\begin{array}{r}1,07 \\
(0,16)\end{array}$ & $\begin{array}{r}0,79 \\
(2,34)\end{array}$ & $\begin{array}{r}1,05 \\
(0,03)\end{array}$ & $\begin{array}{r}1,14 \\
(0,91)\end{array}$ & $\begin{array}{r}0,69 \\
(1,83)\end{array}$ & $\begin{array}{r}2,11^{*} \\
(6,62)\end{array}$ & $\begin{array}{r}0,64 \\
(2,23)\end{array}$ \\
\hline $\begin{array}{l}\text { soziale } \\
\text { Klasse }\end{array}$ & & $\begin{array}{r}1,44^{* *} \\
(13,71)\end{array}$ & $\begin{array}{r}0,99 \\
(0,01)\end{array}$ & $\begin{array}{r}0,97 \\
(0,07)\end{array}$ & $\begin{array}{r}1,04 \\
(0,07)\end{array}$ & $\begin{array}{r}1,13 \\
(2,88)\end{array}$ & $\begin{array}{r}1,15 \\
(1,23)\end{array}$ & $\begin{array}{r}1,26 \\
(1,46)\end{array}$ & $\begin{array}{l}1,05 \\
(0,1)\end{array}$ \\
\hline \multirow{2}{*}{ Bildung } & mittel & $\begin{array}{r}0,72 \\
(1,17)\end{array}$ & $\begin{array}{r}1,06 \\
(0,07)\end{array}$ & $\begin{array}{r}1,54 \\
(2,08)\end{array}$ & $\begin{array}{l}1,03 \\
(0,0)\end{array}$ & $\begin{array}{r}1,57^{*} \\
(6,0)\end{array}$ & $\begin{array}{r}0,78 \\
(0,23)\end{array}$ & $\begin{array}{r}1,11 \\
(0,08)\end{array}$ & $\begin{array}{r}0,86 \\
(0,12)\end{array}$ \\
\hline & hoch & $\begin{array}{r}1,13 \\
(0,13)\end{array}$ & $\begin{array}{r}1,96^{*} \\
(5,72)\end{array}$ & $\begin{array}{r}1,63 \\
(2,31)\end{array}$ & $\begin{array}{r}1,14 \\
(0,07)\end{array}$ & $\begin{array}{r}1,79^{* *} \\
(10,27)\end{array}$ & $\begin{array}{r}1,37 \\
(0,32)\end{array}$ & $\begin{array}{l}1,14 \\
(0,1)\end{array}$ & $\begin{array}{r}0,78 \\
(0,27)\end{array}$ \\
\hline
\end{tabular}


Fortsetzung Tabelle 1

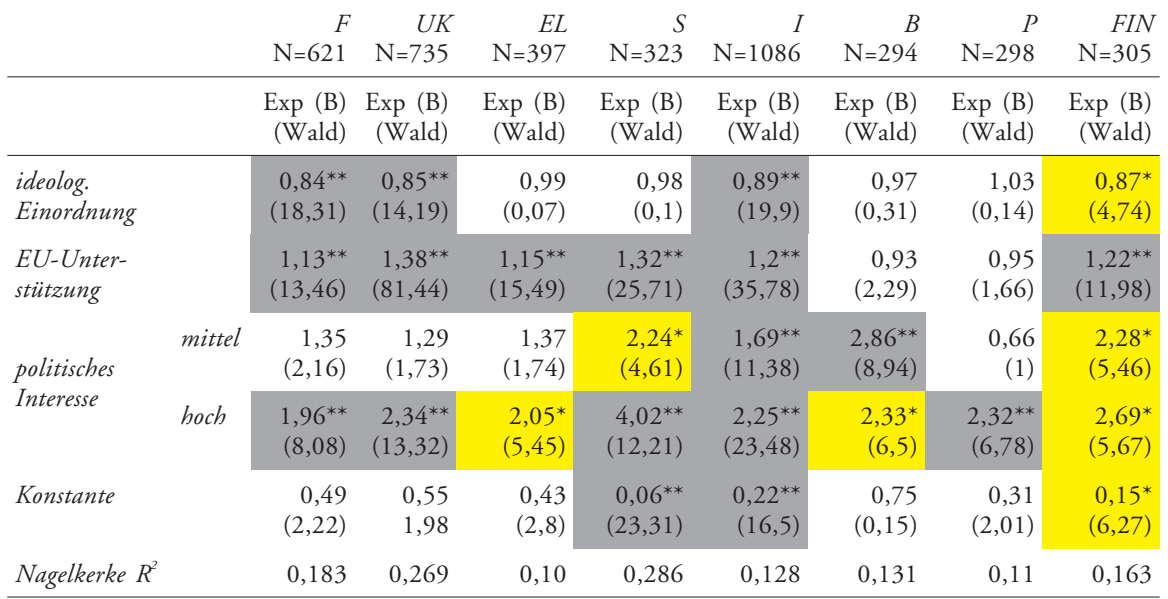

Daten: European Election Study 1999.

Logistische Regression. ${ }^{*} \mathrm{p}<0.05$, hellgrau unterlegt; ${ }^{* *} \mathrm{p}<0.01$, dunkelgrau unterlegt.

Abhängige Variable: Are you, personally, prepared or not to make some personal sacrifice, for example paying a little more taxes, to help another country in the EU experiencing economic difficulties?

Wahrscheinlichkeit beeinflussen, die 1999 in der European Election Study (EES) gestellte Frage nach persönlichen Opfern positiv zu beantworten.

In fast allen Ländern hängt die Bereitschaft, höhere Steuern für ein anderes Land zu akzeptieren, von der Zustimmung zur europäischen Integration ab. Mit jedem Schritt von einer euroskeptischen zu einer eurofreundlichen Haltung steigt die Wahrscheinlichkeit, die EES-Frage positiv zu beantworten. Nur in Belgien und Portugal übt die Bewertung der EU keinen Einfluss aus. In beiden Ländern lehnt die Bevölkerung höhere Steuern zu diesem Zweck mit übergroßer Mehrheit ab. Zudem gilt: Je weiter rechts sich jemand auf der Links-rechts-Skala verortet, desto niedriger ist die Zustimmung zu höheren Steuern (vermutlich unabhängig vom Verwendungszweck); und diejenigen, die sich selbst in ihrer Klassenzugehörigkeit höher einstufen, gehören mit höherer Wahrscheinlichkeit zu den Solidarischen. Schließlich unterstützen die politisch Interessierten und die höher Gebildeten häufiger zwischenstaatliche Umverteilung.

Insgesamt geben die empirischen Befunde den Pessimisten insofern Recht, als nur eine Minderheit der Europäer transnationale Loyalitäten kennt und zur Solidarität bereit ist. Die Mehrheit verankert ihre Zugehörigkeit nach wie vor im Nationalstaat. Prozesse der Gemeinschaftsbildung vollziehen sich, auch ohne den Rekurs auf ethnische Zugehörigkeit, langsam. Neue Loyalitäten sind zudem von der wirtschaftlichen Situation abhängig. Diejenigen, die selbst auf Solidarität angewiesen sind, betrachten die europäische Integration mit Sorge. Grenzöffnung bedeutet für sie eine 
Abbildung 6: Der Beitrag der EU zur Problemlösung

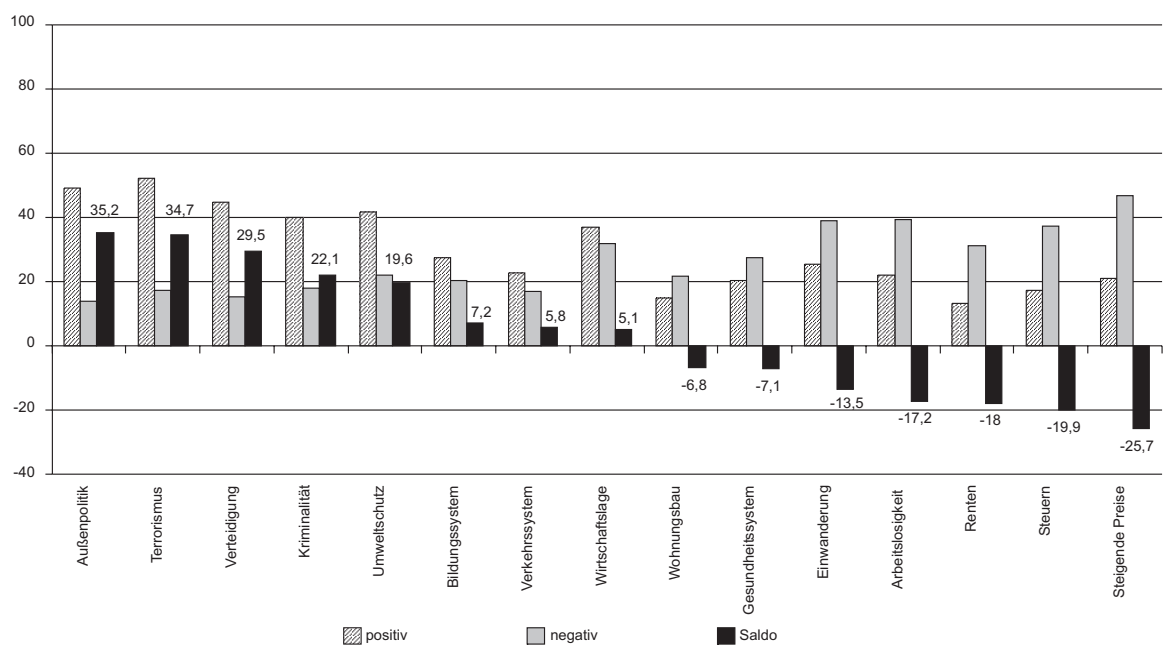

Daten: Eurobarometer 61, 2004, eigene Berechnung.

Entwertung der eigenen Fähigkeiten. Für die Entstehung einer europäischen Identität ist also die Leistungsfähigkeit der EU von besonderer Bedeutung. Die Position der Apologeten und der Fatalisten hängt ganz entscheidend davon ab, dass die EU als wohlfahrtsfördernd wahrgenommen wird. Wie es um die Output-Legitimität der Union bestellt ist, betrachten die nächsten Absätze.

In Umfragen wird regelmäßig die Arbeitslosigkeit als wichtigstes Problem der Mitgliedstaaten genannt. Daran haben die großen Integrationsprojekte der letzten zwei Jahrzehnte wenig geändert, auch wenn das Versprechen auf höheres Wirtschaftswachstum die Notwendigkeit weiterer Integration begründete. Legt man die Erwartung zugrunde, dass die EU als Problemlöser für den Nationalstaat fungieren sollte, enttäuscht die Bilanz. Vom Ziel, der wettbewerbsfähigste Wirtschaftsraum der Welt zu werden, sind die EU-Staaten weit entfernt. Abbildung 6 stellt dar, wie die Bürger die EU-Politik bewerten. Im Kampf gegen die Arbeitslosigkeit und in der Steuer- und Sozialpolitik wird die EU nicht als Problemlöser gesehen, wie dies die Apologeten postulieren, sondern als Problemverschärfer.

Nach den Ängsten befragt, die sie mit der EU-Politik verbinden, nennen fast drei Viertel der Befragten die Angst vor Arbeitsplatzverlagerungen in andere Mitgliedsländer. Diese Befürchtung ist weitgehend unabhängig vom verfügbaren Haushaltseinkommen (Abbildung 7). Auch die Angst, das eigene Land müsse mehr Beiträge zahlen, zieht sich durch alle Länder und Einkommensgruppen. Aber deutlich mehr Menschen mit niedrigem Einkommen fürchten einen Rückgang der Sozialleistungen oder eine Wirtschaftskrise als Folge europäischer Integration. 
Abbildung 7: Angst vor den Folgen der Integration nach Einkommensquartilen gestaffelt

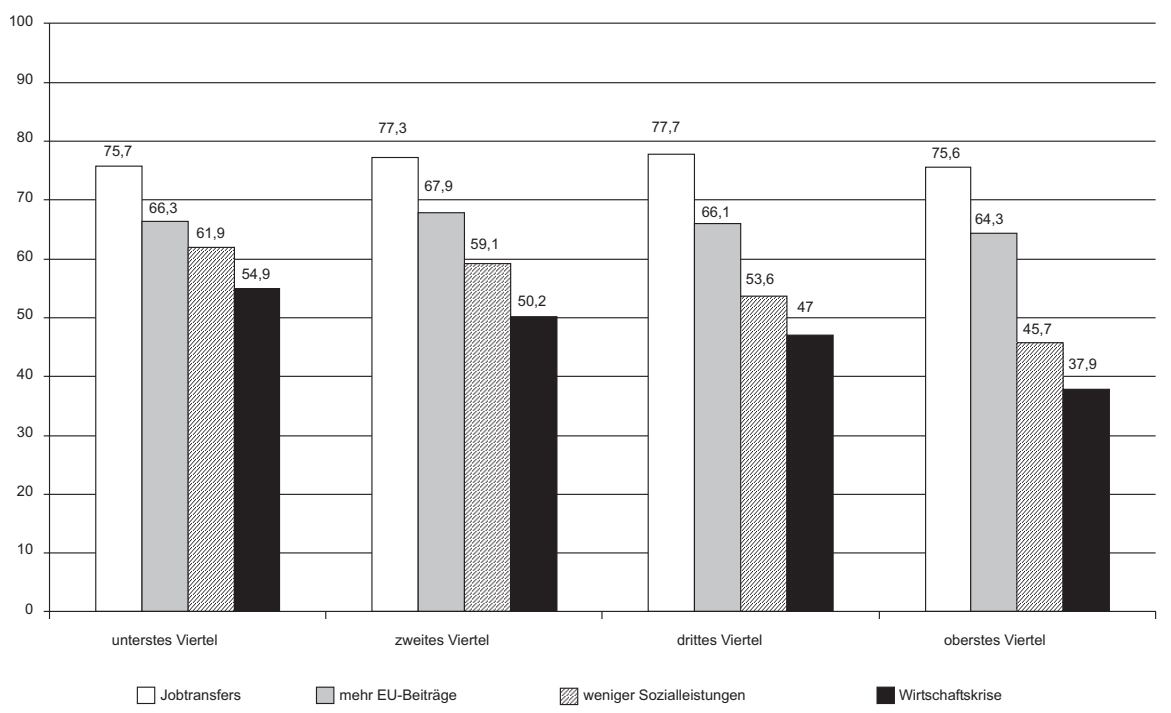

Daten: Eurobarometer 61, 2004, eigene Berechnung.

Während Abbildung 7 auf der wirtschaftlichen Lage der befragten Haushalte beruht, wird der Zusammenhang zwischen den Befürchtungen, die die EU hervorruft, und der persönlichen Situation noch deutlicher, wenn man die persönlichen Erwartungen hinzuzieht (Abbildung 8). Diejenigen, die für die nächsten fünf Jahre eine Verschlechterung ihrer Situation antizipieren, erwarten zu einem besonders hohen Anteil negative Konsequenzen der europäischen Integration. Für einkommensschwache Haushalte verheißt die EU nicht mehr Wohlstand, sondern weniger sozialen Schutz.

In der Wahrnehmung der Bevölkerung verfügt die EU über eine hohe OutputLegitimität in der Außen- und Sicherheitspolitik, aber auch im Umweltschutz und in der Kriminalitätsbekämpfung. Ganz anders sieht dies in der Steuer- und Sozialpolitik aus. Hier wirkt die EU als Bedrohung des nationalen Sozialstaats. Die Daten stützen insofern Majones (2005, S. 36) Forderung, dass die EU nur in Politikbereichen tätig werden sollte, in denen kollektive Güter durch individuelles Handeln bedroht sind, um ihre schmale Legitimitätsbasis nicht zu überreizen. In sensiblen Bereichen des Sozialstaats fehlt ihr für eine eigene Politik die Grundlage. Daraus folgt aber auch, dass die europäische Integration bereits zu weit geht, denn sie verringert die Fähigkeit der Mitgliedstaaten zur Umverteilung. Auch Nicht-Entscheidungen, die redistributiv wirken, müssen legitimiert werden. Das Dilemma der EU besteht darin, dass ihre Legitimität für positive Integration nicht ausreicht, aber der Status quo bereits über verteilungsneutrale Regulierung hinausgeht. Falls die EU in innen- 
Abbildung 8: Persönliche Zukunftserwartung und befürchtete Folgen der Integration

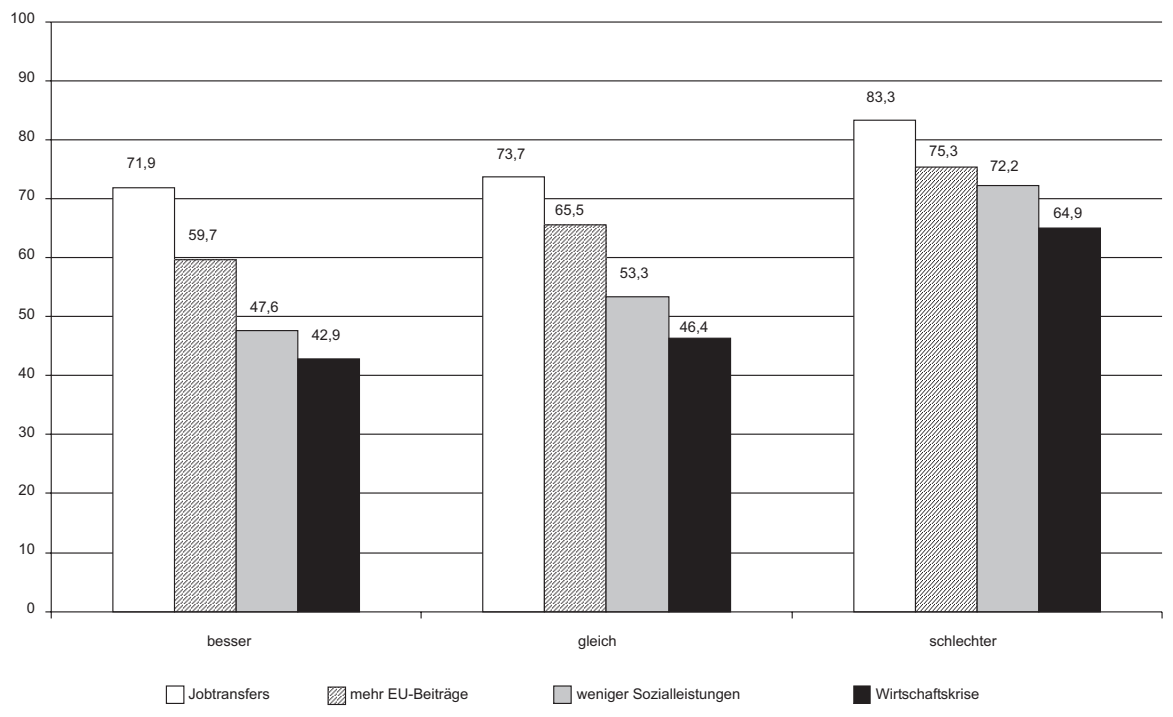

Daten: Eurobarometer 61, 2004, eigene Berechnung.

politischen Auseinandersetzungen über die soziale Sicherung als Konfliktpartei wahrgenommen wird, kann sie nicht länger auf ungeteilte Zustimmung hoffen.

Das Demokratiedefizit der EU wird aus Sicht der Bevölkerung nicht, so lässt sich bilanzieren, über die Output-Seite, also über eine verbesserte Problemlösung aufgehoben. Legitimität hängt auch in der Europäischen Union davon ab, dass die Bürger Einfluss nehmen und die Entscheidungen der Mandatsträger nicht vom der öffentlichen Willensbildung entkoppelt sind. Prinzipiell spricht daher einiges für das Vorhaben, die Bürger über Referenden unmittelbar an politischen Entscheidungen zu beteiligen. Werden diese Abstimmungen in allen Mitgliedstaaten gleichzeitig durchgeführt, könnte eine europaweite Öffentlichkeit entstehen. Der Verfassungsvertrag sieht die Möglichkeit von Bürgerbegehren vor, wenn diese von mindestens einer Million Bürgern aus „einer erheblichen Anzahl von Mitgliedstaaten“ unterstützt werden. Allerdings sind Volksbefragungen in einigen Mitgliedstaaten rechtlich nicht möglich, und die Verfassung ist an zwei Referenden gescheitert. Eine Herausforderung für jede Form direkter Demokratie ist das geringe Wissen der Bürger über die EU. Nach eigener Auskunft wissen 74,9 Prozent der Befragten „ein wenig“ oder „(fast) nichts“ über die Europäische Union. 25 Prozent geben an, „ziemlich“ oder „sehr viel“ zu wissen. Durchschnittlich 47,2 Prozent der Befragten behaupten von sich, die Funktionsweise der EU zu verstehen, fast genauso viele (44 Prozent) sagen 
von sich das Gegenteil (Eurobarometer 62, eigene Berechnung). Die Schwierigkeit anspruchvoller Reformstrategien besteht darin, dass erst durch mehr Bürgerbeteiligung und öffentliche Diskussion das Wissen erzeugt wird, das beide voraussetzen. Verharren die Kenntnisse über die EU-Politik auf niedrigem Niveau, bleibt die deliberative Demokratie ein Elitendiskurs ohne Verankerung in der Bevölkerung.

\section{Schluss}

Mein Artikel hat die Debatte über das EU-Demokratiedefizit aufgegriffen und vier theoretische Positionen - Optimisten, Pessimisten, Apologeten und Fatalisten - dargestellt. Sie unterscheiden sich hinsichtlich der Einschätzung, ob eine Demokratisierung der Europäischen Union möglich und erstrebenswert ist. Für die Optimisten teilt die EU wesentliche Merkmale mit anderen politischen Systemen und kann mit diesen verglichen werden. Eine Demokratisierung der Entscheidungsverfahren ist möglich und wünschenswert. Die Fatalisten interpretieren die EU als bürokratischen konsoziativen Staat, dessen Funktionsfähigkeit von einem intakten Elitenkartell abhängt. „Mehr Demokratie wagen“ hieße, den Bestand der EU zu gefährden. Daher ist aus ihrer Sicht eine Demokratisierung weder wünschenswert noch möglich. Die Pessimisten vergleichen die Staatswerdung der EU mit dem historischen Prozess, der zur Etablierung des Nationalstaats geführt hat. Demokratie und Sozialstaat entstanden in den europäischen Staaten erst, nachdem die Grenzziehung nach außen erfolgt war. Da die europäische Integration Grenzen auflöst, gefährdet sie den sozialen Schutz und die Demokratie in den Mitgliedstaaten. Die Apologten weisen diese Interpretation zurück und argumentieren stattdessen, dass die EU im Kern das Politikversagen der Mitgliedstaaten behebt. Wo diese nicht länger zur eigenständigen Problemlösung in der Lage sind, tritt die Gemeinschaftspolitik an ihre Stelle. Da der Ministerrat weiterhin der zentrale Akteur in der EU-Politik ist, die Delegation an nicht-majoritäre Institutionen dem nationalen Muster folgt und die EU ein „regulativer Staat" ist, wäre eine weitere Politisierung und Parlamentarisierung der EU schädlich.

Die Empirie bestätigt, dass die Bürger die Demokratie in der EU als defizitär wahrnehmen. Sie beurteilen deren Funktionsweise skeptisch und sind mehrheitlich der Meinung, dass ihre Stimme in der EU nicht zählt. Die Aufwertung des Europaparlaments hat den Trend sinkender Wahlbeteiligung nicht gestoppt. In manchen osteuropäischen Ländern gaben weniger als 20 Prozent der Wahlberechtigten bei der Europawahl ihre Stimme ab. Eine weitere Parlamentarisierung der EU erscheint deshalb nicht als probates Mittel gegen das Demokratiedefizit. Gleichfalls skeptisch muss die Strategie bewertet werden, die Legitimität der EU über den Output herzustellen. In der Wirtschafts-, Arbeitsmarkt- und Sozialpolitik wird die Problemlösungsfähigkeit der EU gering eingeschätzt. Menschen mit niedrigem Einkommen und negativen Zukunftserwartungen empfinden die EU als Bedrohung für den so- 
zialen Schutz und die wirtschaftliche Situation. Die Verantwortung für die Sozialund Gesundheitspolitik sollte aus Sicht der Mehrheit weiterhin in den Händen der nationalen Regierung liegen. Eine Vergemeinschaftung dieser Politikfelder findet nur geringe Zustimmung. Positiv wird die Rolle der EU dagegen in der Sicherheitsund Außenpolitik bewertet. Viele Bürger interpretieren die EU nicht als entstehenden Staat, sondern als internationales (außenpolitisches) Regime. Dementsprechend bevorzugt eine Mehrheit intergouvernementale Institutionen, in denen alle Mitgliedstaaten gleichberechtigt sind. Besonders die Bürger kleinerer Staaten wehren sich gegen eine proportionale Stimmengewichtung im Ministerrat.

Einiges spricht für die Interpretation der EU als konsoziativer Staat, der kurzfristig nicht demokratiefähig ist. Genau darin liegt jedoch das Dilemma: Die europäische Integration lässt sich nicht länger als Elite-Projekt fortsetzen, doch gleichzeitig ist der Weg zur europäischen Massendemokratie blockiert. Die EU verharrt in einem Zustand, bei dem immer mehr Entscheidungen umstritten und damit legitimitätsbedürftig sind, aber die Möglichkeit einer weiteren Demokratisierung gering ist. Die gegenwärtige Krise resultiert aus dem Ende des „permissiven Konsenses“, der den Eliten in der Europapolitik weitgehend freie Hand gelassen hatte. Die Referenden der vergangenen Jahre zeigen, dass die Integration nicht länger fraglos als wünschenswert akzeptiert wird (siehe auch schon Reif 1993).

Die Pessimisten warnen zudem, dass der Transfer von Entscheidungskompetenzen auf die EU die nationale Demokratie beschädigen kann. Wenn Regierungen die Verfügungsgewalt über zentrale sozial- und wirtschaftspolitische Entscheidungen abgegeben haben, führen die Parteien im Wahlkampf Scheingefechte. Für die Bürger verliert der Unterschied zwischen den Parteien an Bedeutung, und Wahlen büßen ihre Funktion als Mechanismus der Präferenzaggregation ein. War die europäische Integration als Antwort auf das nationale Politikversagen gedacht, droht der Kompetenztransfer an schwach legitimierte Institutionen in einer Entmündigung der Politik zu enden. Das Scheitern des Verfassungsvertrags und die eingeleitete Denkpause sollten genutzt werden, über eine demokratieschonende Aufgabenteilung zwischen EU-Institutionen und Mitgliedstaaten nachzudenken.

\section{Literatur}

Andeweg, Rudy, 1995: The Reshaping of National Party Systems, in: West European Politics 18, S. 58-78.

Bartolini, Stefano, 2000: Institutional Democratization and Political Structuring in the EU. Lessons from the Nation-state Development, in: Henry Cavanna (Hrsg.), Governance, Globalization and the European Union. Which Europe for Tomorrow?, Dublin: Four Courts Press, S. 129-158.

Bartolini, Stefano, 2005: Restructuring Europe: Centre Formation, System Building and Political Structuring between the Nation-state and the European Union, Oxford: Oxford University Press. 
Chryssochoou, Dimitris N., 1994: Democracy and Symbiosis in the European Union: Towards a Confederal Consociation?, in: West European Politics 17, S. 1-14.

Eberlein, Burkhard und Dieter Kerwer, 2002: Theorizing the New Modes of European Union Governance, in: European Integration online Papers (EIoP) 6.

Eijk, Cees van der und Mark N. Franklin, 2004: Potential for Contestation on European Matters at National Elections in Europe, in: Gary Marks und Marco R. Steenbergen (Hrsg.), European Integration and Political Conflict, Cambridge: Cambridge University Press, S. 32-50.

Eriksen, Erik Oddvar und John Erik Fossum, 2004: Europe in Search of Legitimacy: Strategies of Legitimation Assessed, in: International Political Science Review 25, S. 435-459.

Ferrera, Maurizio, 2005: The Boundaries of Welfare. European Integration and the New Spatial Politics of Social Protection, Oxford: Oxford University Press.

Flora, Peter, 2000: Einführung und Interpretation, in: Stein Rokkan (Hrsg.), Staat, Nation und Demokratie in Europa. Die Theorie Stein Rokkans aus seinen gesammelten Werken rekonstruiert und eingeleitet von Peter Flora, Frankfurt a. M.: Suhrkamp, S. 14-119.

Follesdal, Andreas und Simon Hix, 2005: Why There is a Democratic Deficit in the EU: A Response to Majone and Moravcsik, in: European Governance Papers (EUROGOV) C-05-02. <www.connex-network.org/eruogov/pdf/egp-connec-C-05-02.pdf>

Gabel, Matthew J., 1998: The Endurance of Supranational Governance: A Consociational Interpretation of the European Union, in: Comparative Politics 30, S. 463-475.

Garry, John, Michael Marsh und Richard Sinnott, 2005: „Second-order“ versus „Issue-voting“ Effects in EU Referendums. Evidence from the Irish Nice Treaty Referendums, in: European Union Politics 6, S. 201-221.

Grande, Edgar, 1996: Demokratische Legitimation und europäische Integration, in: Leviathan 24, S. 339-360.

Hix, Simon, Abdul Noury und Gérard Roland, 2005: Power to the Parties: Cohesion and Competition in the European Parliament, 1979-2001, in: British Journal of Political Science 35, S. 209-234.

Hooghe, Liesbet, Gary Marks und Carole J. Wilson, 2004: Does Left/Right Structure Party Positions on European Integration?, in: Gary Marks und Marco R. Steenbergen (Hrsg.), European Integration and Political Conflict, Cambridge: Cambridge University Press, S. $120-140$.

Joerges, Christian und Jürgen Neyer, 1997: From Intergovernmental Bargaining to Deliberative Political Processes: The Constitutionalisation of Comitology, in: European Law Journal 3, S. 273-299.

Kielmansegg, Peter Graf, 2003: Integration und Demokratie, in: Beate Kohler-Koch und Markus Jachtenfuchs (Hrsg.), Europäische Integration, Opladen: Leske + Budrich, S. 49-83.

Klingemann, Hans-Dieter, 2000: Unterstützung für die Demokratie: Ein globale Analyse für die 1990er Jahre, in: Hans-Joachim Lauth, Gert Pickel und Christian Welzel (Hrsg.), Demokratiemessung. Konzepte und Befunde im internationalen Vergleich, Wiesbaden: Westdeutscher Verlag, S. 266-297.

Kneip, Sascha und Alexander Petring, 2006: Die Reformvoschläge der Verfassung für Europa und das Demokratiedefizit der EU, in: Jens Alber und Wolfgang Merkel (Hrsg.), Europas Osterweiterung: Das Ende der Vertiefung. WZB-Jahrbuch 2005, Berlin: edition sigma, S. 207-230.

Kreppel, Amie, 2002: The European Parliament and Supranational Party System: A Study in Institutional Development, Cambridge: Cambridge University Press.

Leibfried, Stephan und Paul Pierson, 1998: Halbsouveräne Wohlfahrtsstaaten: Der Sozialstaat in der Europäischen Mehrebenen-Politik, in: Stephan Leibfried und Paul Pierson (Hrsg.), Standort Europa. Sozialpolitik zwischen Nationalstaat und Europäischer Integration, Frankfurt a.M.: Suhrkamp, S. 58-99. 
Lindberg, Leon N. und Stuart A. Scheingold, 1970: Europe's Would-Be Polity. Patterns of Change in the European Community, Englewood Cliffs, N.J.: Prentice-Hall.

Lipset, Seymour Martin und Stein Rokkan, 1967: Cleavage Structures, Party Systems, and Voter Alignments: An Introduction, in: Seymour M. Lipset und Stein Rokkan (Hrsg.), Party Systems and Voter Alignments: Cross-National Perspectives, New York: The Free Press, S. 1-64.

Mair, Peter, 2001: The Limited Impact of Europe on National Party Systems, in: Klaus H. Goetz und Simon Hix (Hrsg.), Europeanized Politics? European Integration and National Political Systems, London: Frank Cass, S. 27-51.

Mair, Peter, 2005: Popular Democracy and the European Union Polity, in: European Governance Papers (EUROGOV) C-05-03. <www.connex-network.org/euogov/pdf/egp-connex-C-05-03.pdf>

Majone, Giandomenico, 1994: The Rise of the Regulatory State in Europe, in: West European Politics 17, S. 77-101.

Majone, Giandomenico, 2000: The Credibility Crisis of Community Regulation, in: Journal of Common Market Studies 38, S. 273-302.

Majone, Giandomenico, 2005: Dilemmas of European Integration. The Ambiguities and Pitfalls of Integration by Stealth, Oxford: Oxford University Press.

Marks, Gary und Carole J. Wilson, 2000: The Past in the Present: A Cleavage Theory of Party Response to European Integration, in: British Journal of Political Science 30, S. 433-459.

Mau, Steffen, 2003: Wohlfahrtspolitischer Verantwortungstransfer nach Europa? Präferenzstrukturen und ihre Determinanten in der europäischen Bevölkerung, in: Zeitschrift für Soziologie 32, S. 302-324.

Milward, Alan S., 2000: The European Rescue of the Nation-State, 2. Aufl., London: Routledge.

Moravcsik, Andrew, 1997: Warum die Europäische Union die Exekutive stärkt: Innenpolitik und internationale Kooperation, in: Klaus-Dieter Wolf (Hrsg.), Projekt Europa im Übergang? Probleme, Modelle und Strategien des Regierens in der Europäischen Union, Baden-Baden: Nomos, S. 211-269.

Moravcsik, Andrew, 1998: The Choice for Europe. Social Purpose and State Power from Messina to Maastricht, London: UCL Press.

Moravcsik, Andrew, 2002: In Defence of the 'Democratic Deficit': Reassessing Legitimacy in the European Union, in: Journal of Common Market Studies 40, S. 603-624.

Moravcsik, Andrew, 2004: Is there a 'Democratic Deficit' in World Politics? A Framework for Analysis, in: Government and Opposition 39, S. 336-363.

Neyer, Jürgen, 2003: Discourse and Order in the EU: A Deliberative Approach to Multi-Level Governance, in: Journal of Common Market Studies 41, S. 687-706.

Neyer, Jürgen, 2005: Die Krise der EU und die Stärke einer deliberativen Integrationstheorie, in: Zeitschrift für Internationale Beziehungen 12, S. 377-382.

Offe, Claus, 1998: Demokratie und Wohlfahrtsstaat: Eine europäische Regimeform unter dem Streß der europäischen Integration, in: Wolfgang Streeck (Hrsg.), Internationale Wirtschaft, nationale Demokratie. Herausforderungen für die Demokratietheorie, Frankfurt a.M./New York: Campus, S. 99-136.

Olson, Mancur, 1991: Aufstieg und Niedergang von Nationen. Ökonomisches Wachstum, Stagflation und soziale Starrheit, 2. Aufl., Tübingen: J.C.B. Mohr.

Puchala, David, 1972: Of Blind Men, Elephants and International Integration, in: Journal of Common Market Studies 10, S. 267-284.

Reif, Karlheinz, 1993: Ein Ende des 'Permissive Consensus'? Zum Wandel europapolitischer Einstellungen in der öffentlichen Meinung der EG-Mitgliedstaaten, in: Rudolf Hrbek (Hrsg.), Der Vertrag von Maastricht in der wissenschaftlichen Kontroverse, Baden-Baden: Nomos, S. 23-33. 
Rose, Richard und Neil Munro, 2003: Elections and Parties in New European Democracies, Washington: CQ Press.

Scharpf, Fritz W., 2004: Legitimationskonzepte jenseits des Nationalstaats, in: MPIfG Working Paper 04/6.

Schmidt, Manfred G., 2000: Der konsoziative Staat. Hypothesen zur politischen Struktur und zum politischen Leistungsprofil der Europäischen Union, in: Edgar Grande und Markus Jachtenfuchs (Hrsg.), Wie problemlösungsfähig ist die EU? Regieren im europäischen Mehrebenensystem, Baden-Baden: Nomos, S. 33-58.

Taggart, Paul, 1998: A Touchstone of Dissent: Europscepticism in Contemporary Western European Party Systems, in: European Journal of Political Research 33, S. 363-388.

Tsebelis, George und Geoffrey Garrett, 2001: The Institutional Foundations of Intergovernmentalism and Supranationalism in the European Union, in: International Organization 55, S. 357-390.

Weiler, Joseph H. H., Ulrich R. Haltern und Franz C. Mayer, 1995: European Democracy and Its Critique, in: West European Politics 18, S. 4-39.

Zeitlin, Jonathan, 2005: The Open Method of Co-ordination in Action. Theoretical Promise, Empirical Realities, Reform Strategy, in: Johnathan Zeitlin, Philippe Pochet und Lars Magnussen (Hrsg.), The Open Method of Co-ordination in Action. The European Employment and Social Inclusion Strategies, Brussels: P.I.E.-Peter Lang, S. 447-503. 